\title{
Short-Lived Nuclei in the Early Solar System: A Low Mass Stellar Source?
}

\author{
M. Busso ${ }^{1}$, R. Gallino ${ }^{2}$ and G. J. Wasserburg ${ }^{3}$ \\ ${ }^{1}$ Dipartimento di Fisica, Universita' di Perugia, 06123 Perugia, Italy \\ maurizio.busso@ fisica.unipg.it \\ 2 Dipartimento di Fisica Generale, Universita' di Torino and Sezione INFN di Torino, \\ 10125 Torino, Italy \\ gallino@ph.unito.it \\ ${ }^{3}$ The Lunatic Asylum, Division of Geological and Planetary Sciences, \\ CALTECH, Pasadena, CA 91125, USA \\ isotopes@gps.caltech.edu
}

Received 2003 May 4, accepted 2003 July 4

\begin{abstract}
We discuss possible stellar origins of short-lived radioactive nuclei with meanlife $\bar{\tau} \leq 100 \mathrm{Myr}$, which were shown to be alive in the Early Solar System (ESS). We first review current ideas on the production of nuclides having $10 \leq \bar{\tau} \leq 100 \mathrm{Myr}$, which presumably derive from the continuous interplay of galactic astration, nucleosynthesis from massive supernovae and free decay in the interstellar medium. The abundance of the shorter lived ${ }^{53} \mathrm{Mn}$ might be explained by this same scenario. Then we consider the nuclei ${ }^{107} \mathrm{Pd},{ }^{26} \mathrm{Al},{ }^{41} \mathrm{Ca}$ and ${ }^{60} \mathrm{Fe}$, whose early solar system abundances are too high to have originated in this way. Present evidence favours a stellar origin, particularly for ${ }^{107} \mathrm{Pd},{ }^{26} \mathrm{Al}$ and ${ }^{60} \mathrm{Fe}$, rather than an in situ production by energetic solar particles. The idea of an encounter (rather close in time and space) between the forming Sun and a dying star is therefore discussed: this star may or may not have also triggered the solar formation. Recent nucleosynthesis calculations for the yields of the relevant short-lived isotopes and of their stable reference nuclei are discussed. Massive stars evolving to type II supernovae (either leaving a neutron star or a black hole as a remnant) seem incapable of explaining the four most critical ESS radioactivities in their observed abundance ratios. An asymptotic giant branch (AGB) star seems to be a viable source, especially if of relatively low initial mass $\left(M \leq 3 \mathrm{M}_{\odot}\right)$ and with low neutron exposure: this model can provide a solution for ${ }^{26} \mathrm{Al},{ }^{41} \mathrm{Ca}$ and ${ }^{107} \mathrm{Pd}$, with important contributions to ${ }^{60} \mathrm{Fe}$, which are inside the present uncertainty range of the ${ }^{60} \mathrm{Fe}$ early solar system abundance. Such a model requires that ${ }^{26} \mathrm{Al}$ is produced substantially on the AGB by cool bottom processing. The remaining inventory of short-lived species in the solar nebula would then be attributed to the continuous galactic processing, with the exception of ${ }^{10} \mathrm{Be}$, which must reflect production by later proton bombardment at a low level during early solar history.
\end{abstract}

Keywords: solar system: formation — stars: AGB and post-AGB — stars: supernovae: general

\section{Se non è vero è bene trovato}

Ancient Italian Motto

\section{Introduction}

More than forty years ago John Reynolds (1960) at Berkeley announced the discovery of an excess of ${ }^{129} \mathrm{Xe}$ in a meteorite $\left({ }^{129} \mathrm{Xe}^{*}\right)$ and suggested that it could be ascribed to the in situ radioactive decay of ${ }^{129} \mathrm{I}(\bar{\tau}=23$ Myr). This was proven later, through a demonstration that excesses of ${ }^{129} \mathrm{Xe}^{*}$ are directly correlated with stable ${ }^{127}$ I (Jeffery \& Reynolds 1961). These isotopes of iodine are $r$-process products, attributed to supernovae ( $\mathrm{SNe}$ ). It was immediately recognised (Wasserburg, Fowler, \& Hoyle 1960; see also Cameron 1960) that the observed abundance of ${ }^{129}$ I could be ascribed to the long term production of $r$-process nuclei in the Galaxy, provided the solar system material had been isolated from the effects of nucleosynthesis events for about $10^{8}$ years. We can thus recognise how basic questions about the presence of 'live' radioactivities in the Early Solar System (hereafter ESS) were associated with supernova (SN) sources since the beginning of modern research on the subject. However, satisfactory answers for the sources of other nuclei have then been looked for in many works and remain an open issue today.

The search for radionuclides other than ${ }^{129} \mathrm{I}$ was, broadly speaking, not successful for a long period of time. Crucial for all subsequent improvements was the fall of the Allende meteorite in 1969. First of all, this event provided more ${ }^{129} \mathrm{Xe}^{*}$ measurements, and it became possible to sample old materials from the solar nebula including early refractory condensates, the CAIs, or Calcium and Aluminium Inclusions (Podosek \& Lewis 1972). These early-formed samples in Allende also yielded quantitative evidence of the existence of radioactive nuclei with a much shorter meanlife. In particular, ${ }^{26} \mathrm{Al}(\bar{\tau}=1.05 \mathrm{Myr})$ was found to have been present in CAIs with a clear correlation of ${ }^{26} \mathrm{Mg}^{*}$ with ${ }^{27} \mathrm{Al}$ (Lee, Papanastassiou, \& Wasserburg 1976, 1977). Early suggestions by Urey (1955) and Urey \& Donn (1956) indicated that the nuclide ${ }^{26} \mathrm{Al}$, which had just then been discovered (Simanton et al. 1954), was the only reasonable source for the heating and melting of planetesimals.

A few years before the fall of the Allende meteorite, the presence of a relatively long-lived species, ${ }^{244} \mathrm{Pu}$ 
$(\bar{\tau}=115 \mathrm{Myr})$ had been inferred from excesses in neutronrich Xe isotopes (Rowe \& Kuroda 1965). Subsequent work showed $\mathrm{Xe}^{*}$ to be directly associated with excess fission tracks (Cantelaube, Maurette, \& Pellas 1967; Wasserburg, Huneke, \& Burnett 1969a, 1969b; Storzer \& Pellas 1977) with a $\mathrm{Xe}$ isotopic composition showing excesses in $131,132,134,136 \mathrm{Xe}$ identical to that resulting from ${ }^{244} \mathrm{Pu}$ spontaneous fission (Alexander et al. 1971). ${ }^{244} \mathrm{Pu}$-fission Xe was also found in the CAIs from Allende. The hint of excesses of ${ }^{142} \mathrm{Nd}$ found by Lugmair \& Marti (1977) pointed to the possible presence of ${ }^{146} \mathrm{Sm}(\bar{\tau}=148 \mathrm{Myr})$, a $p$-process isotope. Excesses of ${ }^{142} \mathrm{Nd}$ were found to be widespread both in ESS materials and in planetary differentiates and to be well correlated with $\mathrm{Sm}$ (Lugmair et al. 1983; Jacobsen \& Wasserburg 1984; see also Stewart, Papanastassiou, \& Wasserburg 1994). The presence of ${ }^{107} \mathrm{Pd}(\bar{\tau}=9.4 \mathrm{Myr})$ was established by Kelly \& Wasserburg (1978); this nuclide was found to have been present in a large number of iron meteorites representing metal segregation in protoplanets or planetesimals (Chen \& Wasserburg 1981a, 1981b; Carlson \& Hauri 2001).

A wealth of new data subsequently became available on a variety of short-lived nuclei: ${ }^{182} \mathrm{Hf}$ (Harper \& Jacobsen 1994, 1996; Lee \& Halliday 1995, 1996, 1997 , 1998; Yin et al. 2002), ${ }^{41} \mathrm{Ca}$ (Srinivasan et al. 1994, 1996; Sahijpal et al. 1998), ${ }^{53} \mathrm{Mn}$ (Birck \& Allègre 1985; Rotaru et al. 1992; Lugmair \& Shukolyukov 1998), ${ }^{60} \mathrm{Fe}$ (Shukolyukov \& Lugmair 1993a, 1993b) and ${ }^{10} \mathrm{Be}$ (McKeegan et al. 2000). Accounts of some of these findings can be found in reviews by Podosek \& Swindle (1988), Podosek \& Nichols (1997) and Arnould \& Prantzos (1999). The ${ }^{60} \mathrm{Fe}$ is a critical problem which we discuss extensively. Further experimental work and theoretical discussion on various short-lived radioactive isotopes may be found in Hudson et al. (1989), MacPherson, Davis, \& Zinner (1995), Russell et al. (1996), Chen et al. (1996), Murty et al. (1997), Stewart, Papanastassiou, \& Wasserburg (1996), Arnould et al. (1997), Brazzle et al. (1999) and Goswami \& Vanhala (2000).

Recent measurements of ${ }^{26} \mathrm{Al}$ in different $\mathrm{H} 4$ chondrites and a discussion of their use as a fine-scale chronometer can be found in Zinner \& Göpel (2002). These workers sought to establish a relationship between ${ }^{26} \mathrm{Al} /{ }^{27} \mathrm{Al}$ in CAIs and $\mathrm{H} 4$ chondrites and 'absolute' ages (measured backward from today) through the ${ }^{207} \mathrm{~Pb}-{ }^{206} \mathrm{~Pb}$ technique (see also Amelin et al. 2002). As for very fine time resolution, indications have been presented of resolving $\sim 10^{5}$ years in the formation of CAIs by ${ }^{26} \mathrm{Al}$ (Hsu, Wasserburg, \& Huss 2000).

Table 1 summarises the present status of short-lived radioactivities in the ESS. The first column lists the radioactive nuclide $(\mathrm{R})$, the second the stable reference isotope ( $\mathrm{S})$, the third the meanlife of $\mathrm{R}$ and the fourth the ratio $\left(N_{\mathrm{R}} / N_{\mathrm{S}}\right)$ for the initial state of the solar system, as estimated from the measurements. The column labeled 'Place' refers to the class of objects in which evidence has been found for the presence of R. This means that when
Table 1. Short-Lived Radioactivities in the ESS

\begin{tabular}{|c|c|c|c|c|c|}
\hline (R) & (S) & $\begin{array}{l}\text { Mean Life } \\
\quad(\text { Myr) }\end{array}$ & $\left(N_{\mathrm{R}} / N_{\mathrm{S}}\right)_{\mathrm{ESS}}$ & Place $^{a}$ & Ref. $^{b}$ \\
\hline${ }^{10} \mathrm{Be}$ & ${ }^{9} \mathrm{Be}$ & 2.2 & $5.2 \times 10^{-4}$ & CAI & 1 \\
\hline${ }^{26} \mathrm{Al}$ & ${ }^{27} \mathrm{Al}$ & 1.05 & $5.0 \times 10^{-5}$ & $\begin{array}{l}\text { CAI } \\
\mathrm{CH} \\
\mathrm{PD}\end{array}$ & 2 \\
\hline${ }^{41} \mathrm{Ca}$ & ${ }^{40} \mathrm{Ca}$ & 0.15 & $1.5 \times 10^{-8}$ & CAI & 3 \\
\hline${ }^{53} \mathrm{Mn}$ & ${ }^{55} \mathrm{Mn}$ & 5.3 & $1.4 \times 10^{-5}$ & $\begin{array}{l}\text { CAI } \\
\text { PD }\end{array}$ & 4 \\
\hline${ }^{60} \mathrm{Fe}^{c}$ & ${ }^{56} \mathrm{Fe}$ & 2.2 & $\begin{aligned} 1.6 & \times 10^{-8} \\
\sim & \sim 10^{-6} \\
3 & \times 10^{-7} \\
-2 & \times 10^{-6}\end{aligned}$ & $\begin{array}{l}\text { PD } \\
\text { CAI } \\
\text { UOC }\end{array}$ & $\begin{array}{l}5 \\
6 \\
7\end{array}$ \\
\hline${ }^{107} \mathrm{Pd}$ & ${ }^{108} \mathrm{Pd}$ & 9.4 & $\simeq 2.0 \times 10^{-5}$ & PD & 8 \\
\hline${ }^{129} \mathrm{I}$ & ${ }^{127} \mathrm{I}$ & 23 & $1.0 \times 10^{-4}$ & $\begin{array}{l}\text { CAI } \\
\text { CH }\end{array}$ & 9 \\
\hline${ }^{146} \mathrm{Sm}$ & ${ }^{144} \mathrm{Sm}$ & 148 & $7.1 \times 10^{-3}$ & PD & 10 \\
\hline${ }^{182} \mathrm{Hf}$ & ${ }^{180} \mathrm{Hf}$ & 13 & $2.0 \times 10^{-4}$ & $\begin{array}{l}\mathrm{PD} \\
\mathrm{CH}\end{array}$ & 11 \\
\hline${ }^{244} \mathrm{Pu}$ & ${ }^{232} \mathrm{Th}$ & 115 & $3.0 \times 10^{-3}$ & $\begin{array}{l}\text { CAI } \\
\text { PD }\end{array}$ & 12 \\
\hline${ }^{36} \mathrm{Cl}^{d}$ & ${ }^{35} \mathrm{Cl}$ & 0.43 & $1.4 \times 10^{-6}$ & CAI & 13 \\
\hline${ }^{135} \mathrm{Cs}^{d}$ & ${ }^{133} \mathrm{Cs}$ & 2.9 & $1.6 \times 10^{-4}$ & CAI & 14 \\
\hline${ }^{247} \mathrm{Cm}^{d}$ & ${ }^{232} \mathrm{Th}$ & 23 & $<6 \times 10^{-4}$ & CAI & 15 \\
\hline
\end{tabular}

${ }^{a}$ Place - indicates the type of sample from which the data were derived $\mathrm{CAI}$ refers to Calcium-Aluminium Inclusions; $\mathrm{CH}$ refers to phases or assemblages in chondrites but not to those in CAI; PD indicates planetary differentiates (major FeNi-metal segregations or magmatic rocks, e.g. basalts). UOC is used for Unequilibrated Ordinary Chondrites. For ${ }^{26} \mathrm{Al}$ there is evidence of its presence in $\mathrm{CH}$ and rarely in $\mathrm{PD}$ at abundances far below the ESS value. For ${ }^{53} \mathrm{Mn}$ there is a suggestion in CAIs and, at a somewhat lower level, in PD. For ${ }^{60} \mathrm{Fe}$, the first value quoted follows discussion by Lugmair, Shukolyukov, \& MacIsaac (1995).

${ }^{b}$ References - (1) McKeegan, Chaussidon, \& Robert (2000); (2) Lee et al. (1976, 1977); (3) Srinivasan, Ulyanov, \& Goswami 1994, Srinivasan et al. 1996; (4) Birck \& Allègre (1985); Rotaru, Birck, \& Allègre (1992); Lugmair \& Shukolyukov (1998); Hutcheon et al. (1998); (5) Shukolyukov \& Lugmair (1993a, 1993b); Lugmair et al. (1995); (6) Birck \& Lugmair 1988; (7) Tachibana \& Huss (2003a, 2003b), Mostefaoui et al. (2003); (8) Kelly \& Wasserburg (1978); (9) Reynolds (1960); Jeffery \& Reynolds (1961); Brazzle et al. (1999); (10) Lugmair \& Marti (1977); Lugmair \& Galer 1992; (11) Harper \& Jacobsen (1994); Lee \& Halliday (1995); Yin et al. (2002); Kleine et al. (2002); (12) Rowe \& Kuroda (1965); Alexander et al. (1971); (13) Murty, Goswami, \& Shukolyukov (1997); (14) McCulloch \& Wasserburg (1978); (15) Chen \& Wasserburg 1981a, 1981b.

${ }^{c}$ Estimates for different samples are distributed over two orders of magnitude. However, recent data, derived from Unequilibrated Ordinary Chondrites (UOC), cover a smaller range (one dex).

${ }^{d}$ In the lower panel we include isotopes for which only hints or upper bounds are available.

'Place' refers to very early condensates, like CAIs, then the measurement and the data for the initial state (column 4) coincide. When, instead, measurements refer to younger samples, such as, for example, ${ }^{107} \mathrm{Pd}$, then the initial abundance should be higher than measured. The initial value depends on estimates for the sample age and on the meanlife of the isotope. From consideration of the observations referred to here, the radionuclides listed in 
the upper panel were all present in the early solar system. The daughter products associated with these nuclei are not the result of decay prior to formation of the solar system as argued extensively by some workers, but in fact represent decay of the radionuclide within the solar system. Most of the short-lived nuclei that were present in the ESS and are discussed here had to be transported in from the interstellar medium (ISM). The carrier grains responsible for the bulk solar inventory of these nuclei have not been identified. Clear evidence of extinct radionuclides in presolar grains, which decayed prior to their incorporation, has now been established (see also Zinner 1998) but that is a separate issue. Some of these types of grains must have been the carriers of the short-lived nuclei.

From the point of view followed here, the abundances of the radionuclides in the ESS are taken to represent the bulk solar system values at the moment of solar system formation. If this is not true, then the problem is fundamentally different from what is discussed here. The return of cometary samples from the stardust mission should establish just how representative the values used here are. They then should represent freshly synthesised nuclei (depending on their respective $\bar{\tau}$ ) incorporated from the ISM when the solar system formed. Alternatively, some of them may have been produced in the solar system during an early $\mathrm{T}$ Tauri phase of the Sun, a topic that will be referred to later. In our approach, the value of $N_{\mathrm{R}} / N_{\mathrm{S}}$ inferred for a particular sample is simply related to the initial value for an approximately homogenised protosolar cloud at some initial time $(t=0)$ corrected for radioactive decay by $\exp (-t / \bar{\tau})$ until the time $(t)$ of formation of the particular sample. Note that the inferred presence of a radionuclide in a planetary differentiate (samples of a planet that was physically segregated at some time after melting and crystallisation) requires that the body formed sufficiently early. The issue is: what is that time? For a chondrite, which is an aggregate of very diverse objects, there are several questions to be addressed. In what sub-object, phase, or inclusion is evidence of the radionuclide preserved? How did these sub-objects form? What is their connection to the bulk solar material? Insofar as the formation times are much shorter than $\bar{\tau}$, then the observed ratio should nearly represent the value of the 'initial' state. This is the case for the more long-lived ${ }^{244} \mathrm{Pu}$ and ${ }^{146} \mathrm{Sm}$. For the case of ${ }^{129} \mathrm{I}$, the measurement in chondrites and in CAIs (showing volatile element alteration) are all at the level of $1.0 \times 10^{-4}$ and should represent the initial state. For the case of ${ }^{107} \mathrm{Pd}$, the ratio ${ }^{107} \mathrm{Pd} /{ }^{108} \mathrm{Pd}$ of about $2 \times 10^{-5}$ is typical of many $\mathrm{Fe}-\mathrm{Ni}$ meteorites. Insofar as the time scale for proto-planet formation is not larger than a few million years, these values should also be close to the initial solar inventory (within a factor of two) and have therefore been adopted in Table 1 .

For the critical case of ${ }^{26} \mathrm{Al}$, we note that the highest value observed in a large number of CAIs for ${ }^{26} \mathrm{Al} /{ }^{27} \mathrm{Al}$ is about $5 \times 10^{-5}$ (see review by MacPherson et al. 1995). However, many CAIs show lower values, ranging down to zero. This has been interpreted as the result of either later formation of some CAIs (up to a few million years) or of auto-metamorphism resulting from the intense ${ }^{26} \mathrm{Al}$ heat source in small planetesimals (LaTourrette \& Wasserburg, 1997). Evidence of ${ }^{26} \mathrm{Al}$ has been found at intermediate abundance levels in glasses present in chondrules (in unmetamorphosed chondrites) and small excesses of ${ }^{26} \mathrm{Mg}$ correlated with $\mathrm{Al}$ have recently been identified in some eucrites which are basaltic planetary differentiates (Srinivasan et al. 1996). These data appear to make a selfconsistent story. We then take the value for ${ }^{26} \mathrm{Al} /{ }^{27} \mathrm{Al}$ of $5 \times 10^{-5}$ to represent something like the original inventory of the protosolar nebula and consider the lower values in solar system processed material to represent the passage of time and metamorphism. Some preserved circumstellar grains from stars which existed before the sun show ${ }^{26} \mathrm{Al} /{ }^{27} \mathrm{Al}$ ratios several orders of magnitude above the value of ${ }^{26} \mathrm{Al} /{ }^{27} \mathrm{Al}$ in the solar nebula.

There are three other cases that require special attention. These are ${ }^{41} \mathrm{Ca}$ (with a much shorter meanlife, $\bar{\tau}$, of $0.15 \mathrm{Myr}),{ }^{10} \mathrm{Be}(\bar{\tau}=2.2 \mathrm{Myr}$, not produced in stellar sources) and ${ }^{60} \mathrm{Fe}(\bar{\tau}=2.2 \mathrm{Myr})$, whose abundance in the ESS is still uncertain (see Table 1). These are discussed in later sections.

In general, the origins of the nuclei in Table 1 are a matter of large debate. They include the continuous synthesis/destruction in the Galaxy by different stellar sources (Schramm \& Wasserburg 1970), a local pollution by a nearby star (Cameron \& Truran 1977; Wasserburg et al. 1994) and, for certain species, the in situ production in some part of the accretion disk through spallation processes by local cosmic rays or in the $\mathrm{X}$-winds of the forming Sun during its T Tauri stage. These processes were recognised earlier and have recently been intensely investigated (Shu, Shang, \& Lee 1996; Shu et al. 1997, 2001). Associated with these works there is also a recent suggestion for production of some species $\left({ }^{10} \mathrm{Be}\right.$ in particular) in cosmic rays (Desch, Srinivasan, \& Connolly 2003). Uncertainties in the interpretations are due to the still poorly known intricacies of stellar nucleosynthesis mechanisms (particularly for the $r$-process), of magnetohydrodynamics in the environments around newly formed stars and of the precursors and formation mechanisms of chondritic materials.

In this paper we present the status of these researches, together with new calculations on short-lived isotope production in AGB stars. A critical issue is the production of ${ }^{60} \mathrm{Fe}$ and its abundance in the ESS. We first review, in Section 2, the present knowledge of the galactic inventory of short-lived radioactive nuclei, as derived from uniform nucleosynthesis processes through the galactic lifetime and as reflected in the abundance of some of them in the ESS. In Section 3 we broadly consider the alternative ideas on 'local' production sites for the short-lived nuclei that cannot be accounted for by the uniform galactic production. In Section 4 the more specific case of a nearby stellar source is illustrated and previous results are updated by discussing recent calculations for massive stars and by presenting our own new set of nucleosynthesis models for 
AGB stars of different masses. In this context we show that a late pollution from a relatively low-mass AGB star (Wasserburg et al. 1994, 1995) still appears today as a possible model for explaining the ESS inventory of ${ }^{26} \mathrm{Al}$, ${ }^{41} \mathrm{Ca},{ }^{60} \mathrm{Fe}$ and ${ }^{107} \mathrm{Pd}$.

\section{Galactic Inventory of Short-Lived Nuclei}

\subsection{Uniform Production in the Galaxy as Inferred from ESS Data}

The age of the solar system has been ascertained to be $4.566 \times 10^{9}$ yr (Bahcall, Pinsonneault, \& Wasserburg 1995; Allègre et al. 1995). The uncertainty may be less than 2 Myr (see Tera \& Carlson 1999; Amelin et al. 2002). The most 'precise' ages are obtained using the so-called ${ }^{207} \mathrm{~Pb}-{ }^{206} \mathrm{~Pb}$ method. The ages of solar system objects are determined from the measured abundances of longlived parent nuclei today and of their accumulated stable daughter products in meteorites. This method is applied to unstable nuclei still preserved now and requires that their meanlife is sufficiently long. The shortest lived nucleus to which this rule applies is ${ }^{235} \mathrm{U}\left(\bar{\tau}=1.015 \times 10^{9} \mathrm{yr}\right)$. All nuclei with a meanlife significantly shorter than this are now effectively extinct, due to exponential decay: $\exp \left(-4.566 \times 10^{9} / \bar{\tau}\right)$. The original presence of shorter lived nuclei can only be inferred from their decay products as the parent isotopes are absent. In many cases, their ESS concentration was inherited from the abundance established in the interstellar medium as a balance between stellar production and decay. When this occurs, one can easily estimate the equilibrium ratio $N_{\mathrm{R}} / N_{\mathrm{S}}$ for the short-lived radioactive nucleus $\mathrm{R}$ with respect to a stable isotope $S$ produced in the same process. In the simplest possible galactic model (a closed box, with instantaneous recycling, evolving for a time duration $T$ ) this ratio is (Schramm \& Wasserburg 1970):

$$
\left[N_{\mathrm{R}}(T) / N_{\mathrm{S}}(T)\right]_{\mathrm{UP}} \simeq P_{\mathrm{R}} p(T) \bar{\tau}_{\mathrm{R}} /\left(P_{\mathrm{S}}\langle p\rangle T\right)
$$

Here UP means uniform production (in the galaxy), $P_{\mathrm{S}}\langle p\rangle$ is the stellar production rate of the stable nucleus, expressed as the product of an assumed constant stellar production factor $P_{\mathrm{S}}$ and of the average effective production rate $\langle p\rangle$ over $T$, where $p$ is a function of time, $P_{\mathrm{R}}$ is the stellar production factor for the radioactive isotope and $p(T)$ is the effective rate at time $T$ when production ceased. If the solar system material is separated from the interstellar medium for a certain time interval $\Delta_{1}$ before forming the first solid condensates (due to the collapse time of the parent cloud core for example), then the abundance ratio $N_{\mathrm{R}} / N_{\mathrm{S}}$ in the oldest meteoritic material will be decreased with respect to the galactic equilibrium by a factor of $\exp \left(-\Delta_{1} / \bar{\tau}_{\mathrm{R}}\right)$. We identify the oldest solar system material with CAIs. Any planetary differentiate formed after a further time delay $\Delta_{2}$ will have an abundance ratio further reduced by $\exp \left(-\Delta_{2} / \bar{\tau}_{\mathrm{R}}\right)$. These early abundances can be established today from the decay products, after a careful demonstration that their excess is quantitatively correlated with the chemical properties of the parent element, not with the daughter element. Descriptions of the procedure can be found in Lee et al. (1976) and in Wasserburg (1987). Note that excesses or deficiencies in the abundance of an isotope may hint at the presence of a radioactive precursor. However, this may also be due to the widespread intrinsic isotopic heterogeneity in the early solar system that is now well established at the level of a few parts in $10^{4}$. It is the correlation of excesses of a nuclide with a supposed parent element in different phases that were formed at the same time from a homogeneous source that is required to demonstrate the presence and abundance of a radioactive parent.

In the above framework, it was argued that ${ }^{244} \mathrm{Pu}$ (an actinide produced solely by the $r$-process and with a relatively long meanlife) has an ESS abundance approximately compatible with the uniform production in the Galaxy over a time of around $10^{10} \mathrm{yr}$ (Wasserburg, Busso, \& Gallino 1996). A similar compatibility exists for several purely $r$-process nuclei with mean lives larger than a few Myr, as noted by several authors, among them Cameron (1993), Cameron, Thielemann, \& Cowan (1993), Cameron et al. (1995) and Meyer \& Clayton (2000). In particular, Wasserburg et al. (1996) noted that an isolation time $\Delta \simeq 10^{7} \mathrm{yr}$ would allow one to grossly explain, through the continuous process of galactic enrichment by the same $r$-process type source (assumed to be a supernova), the ESS abundances of actinides and those of the shorter lived ${ }^{182} \mathrm{Hf}$ and ${ }^{146} \mathrm{Sm}$. This assumes that the $p$-nuclide ${ }^{146} \mathrm{Sm}$ is co-produced in $r$-process events. When specific supernova models were considered, such as that of a $25 \mathrm{M}_{\odot} \mathrm{SN}$ by Woosely \& Weaver (1995), it turned out that ${ }^{53} \mathrm{Mn}$, despite its rather short meanlife ( $\bar{\tau}=5.3 \mathrm{Myr}$ ), could also be ascribed to the continuous injection of supernova material into the ISM (Wasserburg et al. 1996; Meyer \& Clayton 2000). However, as the time scale of about $10^{7} \mathrm{yr}$ is much shorter than the interval of $\sim 10^{8}$ yr inferred for ${ }^{129} \mathrm{I}$, this scenario would overestimate the abundance of ${ }^{129} \mathrm{I}$ by a large factor. It was therefore proposed that iodine, together with all $r$-nuclei below the atomic mass number $\mathrm{A} \sim 140$, be ascribed to a different supernova type than the one responsible for heavier nuclei. ESS abundances thus appear to require that the SN explosions contributing iodine must be much rarer events, so that the solar nebula would be isolated from their contributions for about $7 \times 10^{7} \mathrm{yr}$. This fact, noted much earlier (Wasserburg et al. 1960; Schramm \& Wasserburg 1970; Cameron et al. 1993, 1997), led to a series of works, both theoretical and observational, on the ensuing bimodal or multiple nature of the $r$-process source types (for example, see Wasserburg et al. 1996; Qian, Vogel, \& Wasserburg 1998a, 1998b). Further, it has been found that at low metallicities the observed abundances of heavy $r$-process nuclei in halo stars are very similar to the solar $r$-process pattern (Sneden et al. 1998; Cowan et al. 1999) but that the light nuclei show significant deficiencies (see Sneden et al. 2000). It follows that a single, prototypical $r$-process source (which has long been the focus of attention) is no 
longer acceptable (Wasserburg, Gallino, \& Busso 1998; Qian \& Wasserburg 2003).

Observations of low metallicity stars also suggest that the stellar sites for production of heavy $r$-process nuclei do not coincide with those producing $\mathrm{O}$ through $\mathrm{Zn}$, including the Fe peak elements (Travaglio et al. 1999; Qian \& Wasserburg 2002). They are thus not produced in SNe II (supernovae type II) with $M \geq(10-12) \mathrm{M}_{\odot}$. It has been proposed (Cohen et al. 2003; Qian \& Wasserburg 2003) that neutron star formation by accretion induced collapse in binary systems may be the source of heavy $r$ nuclei. Thus, while the sources of heavy $r$ nuclei are a matter of active investigation, the usual single source model for all $r$ nuclei is not viable.

\subsection{Present Galactic Inventory of ${ }^{26} \mathrm{Al}$ and ${ }^{60} \mathrm{Fe}$}

The process of uniform galactic production discussed in the previous subsection is considerably insufficient to account for the abundance of a few shorter-lived nuclei, including ${ }^{26} \mathrm{Al}$ and ${ }^{60} \mathrm{Fe}$. The ongoing synthesis of these two isotopes can, in principle, be tested independently through observations, thanks to the fact that their decay is accompanied by the emission of gamma-ray lines (at $1.809 \mathrm{MeV}$ for ${ }^{26} \mathrm{Al}$ and at 1.173 and $1.332 \mathrm{MeV}$ for ${ }^{60} \mathrm{Fe}$, from the decay of the daughter ${ }^{60} \mathrm{Co}$ ). The ${ }^{26} \mathrm{Al} \gamma$-line in the galaxy was first found by Mahoney et al. (1984) using detectors on HEAO (High Energy Astrophysical Observatory). Extensive measurements were made for these nuclei by $\gamma$-ray line instruments (for example COMPTEL, the Compton Gamma Ray Observatory, and GRIS, the balloon-borne Gamma Ray Imaging Spectrometer). These observations were reviewed in a number of papers (see Diehl \& Timmes 1996; Naya et al. 1998; Prantzos \& Diehl 1996 and references therein). Recent results from these experiments, assuming a steady-state production, suggest an average abundance of ${ }^{26} \mathrm{Al}$ in the Galaxy of $3.1 \pm 0.9 \mathrm{M}_{\odot}$ (COMPTEL) and in the range from $2.6 \pm 0.4$ to $4.5 \pm 0.7 \mathrm{M}_{\odot}$ (GRIS). This distribution of ${ }^{26} \mathrm{Al}$ sources is clumpy, certainly not uniform. The total amount of galactic ${ }^{26} \mathrm{Al}$ corresponds to an average ${ }^{26} \mathrm{Al} /{ }^{27} \mathrm{Al}$ in the ISM of a few times $10^{-6}$. This is a factor of 10-25 less than the value in CAIs, indicating that the solar system initially had a much higher enhancement of ${ }^{26} \mathrm{Al}$ (due to the protosolar cloud being closer to the source). Recent models for massive star evolution suggest a value $2.2 \pm 0.4 \mathrm{M}_{\odot}$ as coming from massive star progenitors (Knödlseder 1999). The theoretical and observational estimates are only marginally compatible and a further $1 \mathrm{M}_{\odot}$ of ${ }^{26} \mathrm{Al}$ from undetected, low-efficiency or dispersed sources cannot be excluded; this might actually improve the agreement (Lentz, Branch, \& Baron 1999). Although most galactic ${ }^{26} \mathrm{Al}$ certainly comes from massive stars, either from exploding type II SNe (Rauscher et al. 2002; Chieffi \& Limongi 2003) or from the winds of Wolf-Rayet stars (Meynet et al. 1997; Arnould et al. 1997), these stars might not be sufficiently numerous to account for the whole galactic inventory and some contribution might come from AGB stars (Busso, Gallino, \& Wasserburg 1999; Mowlavi \& Meynet 2000; Nollett, Busso, \& Wasserburg 2003) and novae (Starrfield et al. 2000; Iliadis et al. 2002).

The nuclide ${ }^{60} \mathrm{Fe}$ has not been detected so far, neither by COMPTEL nor by GRIS. Massive star nucleosynthesis predicts this nuclide to come from almost the same stellar zones as ${ }^{26} \mathrm{Al}$, and, in many cases, its yield appears to be smaller by only a factor of 2-3 (for example, see Meyer \& Clayton 2000). A rough rule of thumb is that if only massive stars produce the galactic abundance of both nuclei, then the similar yields and the longer meanlife of ${ }^{60} \mathrm{Fe}$ should guarantee that the $\gamma$-ray flux for this isotope be rather close to the present detection limits, whenever ${ }^{26} \mathrm{Al}$ is detected. A further contribution to ${ }^{60} \mathrm{Fe}$ might come from supernovae of Type Ia (Timmes et al. 1995). Available data provide only an upper limit to the flux ratio, yielding ${ }^{60} \mathrm{Fe} /{ }^{26} \mathrm{Al} \lesssim 0.14$ (Naya et al. 1998). This is far below the values expected from recent SN II models (see below).

\section{Models for the Production of Short-Lived Nuclei}

\subsection{Exotic or Local Origin?}

While most of the short-lived nuclei in the ESS listed in Table 1 are certainly a result of stellar nucleosynthesis processes, models of spallation reactions have suggested that the fast particles ejected by the Sun in its T Tauri phase may provide a source for a few species, especially ${ }^{10} \mathrm{Be},{ }^{26} \mathrm{Fe},{ }^{41} \mathrm{Ca}$ and ${ }^{53} \mathrm{Mn}$ (Shu et al. 1997; Lee et al. 1998; Gounelle et al. 2001). In these models the hypothesis was advanced that CAIs and chondrules are also produced in the so-called $\mathrm{X}$-winds from the early Sun. Hence, the high abundance measured in CAIs for ${ }^{26} \mathrm{Al}\left({ }^{26} \mathrm{Al} /{ }^{27} \mathrm{Al}=5 \times 10^{-5}\right)$ by Lee et al. (1977) would not be indicative of a widespread distribution of this nuclide in the protosolar cloud. In this case the many properties attributed to ${ }^{26} \mathrm{Al}$ would have to be reconsidered, from its use as a chronometer to its relevance for melting the protoplanetary material (Urey 1955; Fish, Goles, \& Anders 1960; Schramm, Tera, \& Wasserburg 1970). It has long been known that proton bombardment of bulk solids in the ESS would readily produce ${ }^{53} \mathrm{Mn}$ but greatly underproduce ${ }^{26} \mathrm{Al}$ (Wasserburg \& Arnould 1987). The issue at hand for 'local origin' is to seek a self-consistent solution for several short-lived nuclides at the appropriate observed abundances in a physically consistent scenario. In this respect, a model calculation that has attracted a great deal of attention is that by Gounelle et al. (2001) in which the authors were able to predict ${ }^{10} \mathrm{Be},{ }^{26} \mathrm{Al},{ }^{41} \mathrm{Ca}$ and much of the ${ }^{53} \mathrm{Mn}$ inferred for CAIs through irradiation of fast particles from the early Sun. The model requires that the irradiation takes place in low-energy impulsive flares in which ${ }^{3} \mathrm{He}$ is enhanced by several orders of magnitude relative to $\mathrm{H}$ in the cosmic rays. The low energy of the cosmic rays and the high abundance of ${ }^{3} \mathrm{He}$ allows the production of ${ }^{26} \mathrm{Al}$ to be pumped up, which in turn suppresses production of Be. However, the energy spectrum and ${ }^{3} \mathrm{He}$ 
abundance required for this model to work are at the very extreme limits of those observed in impulsive flares in the current Sun. In order to make the scheme also work for ${ }^{41} \mathrm{Ca}$, a layered structure of target objects is required. In general, production of the nuclei considered by a series of solar cosmic ray bombardments appears to be possible in principle. This, however, can only be achieved under extreme, and probably implausible, conditions; furthermore, the $\mathrm{X}$-wind model does not produce ${ }^{107} \mathrm{Pd}$. We consider the proposal by Gounelle et al. (2001) to be an interesting but rather ad hoc model.

Moreover, recent experimental evidence does not support the X-wind source scenario. The presence of ${ }^{26} \mathrm{Al}$ in a highly volatile Na-rich glass in chondrules is not compatible with this source (Russell et al. 1996) nor is this compatible with the observation of small ${ }^{26} \mathrm{Al}$ effects in planetary differentiates (Srinivasan et al. 1994, 1996; Marhas et al. 2000). After the discovery by McKeegan et al. (2000) of ${ }^{10} \mathrm{Be}$, measurements by MacPherson and Huss (2001) and by Marhas, Goswami, \& Davis (2002) provided evidence for the presence of ${ }^{10} \mathrm{Be}$ in meteoritic material in CAIs that is not correlated with ${ }^{26} \mathrm{Al}$ and in much younger material (for example, planetary differentiates) in which no live ${ }^{26} \mathrm{Al}$ or ${ }^{41} \mathrm{Ca}$ was present (see also MacPherson, Huss, \& Davis 2003). This implies that ${ }^{10} \mathrm{Be}$ was formed after ${ }^{26} \mathrm{Al}$ and ${ }^{41} \mathrm{Ca}$ had decayed. The presence of ${ }^{53} \mathrm{Mn}$, evidence for which is clearly found in planetary differentiates, also does not seem to support the X-wind model (Lugmair \& Shukolyukov 1998). Also, ${ }^{60} \mathrm{Fe}$ is clearly not produced in this way (Tachibana \& Huss 2003a), so that the early X-wind model or local cosmic ray sources (Desch et al. 2003) remain as open and plausible scenarios only for ${ }^{10} \mathrm{Be}$, which in turn is not produced by stellar nucleosynthesis (for example, see Pagel 1997). Similar conclusions as presented here were drawn by several other workers (see Zinner \& Göpel 2002). Measurements indicating the absence of ${ }^{26} \mathrm{Al}$ in samples with ${ }^{10} \mathrm{Be}$ seem to rule out the plausibility of recent models of the ejection of supernova material in jets (Cameron 2001), as production of ${ }^{10} \mathrm{Be}$ would be accompanied by ${ }^{26} \mathrm{Al}$ and ${ }^{41} \mathrm{Ca}$ in these models, yielding a correlation between the three nuclides that is not seen. On the other hand, estimates of the absolute age (measured back from the present) of chondrules and CAIs through the $\mathrm{Pb}-\mathrm{Pb}$ technique (Amelin et al. 2002) yielded age differences consistent with the different ${ }^{26} \mathrm{Al} /{ }^{27} \mathrm{Al}$ values, again supporting the original interpretation of ${ }^{26} \mathrm{Al}$ data as a chronometer of ESS materials. The ${ }^{10} \mathrm{Be}$ data clearly show that some proton bombardment took place in the ESS, but probably this occurred at a level insufficient to coproduce the other short lived nuclei.

\subsection{Is a Close Stellar Encounter Plausible?}

The requirement for a nearby stellar source contributing freshly synthesised material to the protosolar cloud seems unavoidable. The possibility of a local contamination by a nearby star was considered by many authors, after the proposal of the supernova trigger advanced by Cameron \& Truran (1977). The reader may find more discussion of this model in Podosek \& Nichols (1997). To obtain an ${ }^{26} \mathrm{Al} /{ }^{27} \mathrm{Al}$ ratio of $\sim 5 \times 10^{-5}$ in the protosolar cloud would require that about $5 \%$ of all the stable ${ }^{27} \mathrm{Al}$ in the ESS be from a close-by SN source, provided that ${ }^{26} \mathrm{Al} /{ }^{27} \mathrm{Al}$ of $\sim 10^{-3}$ is typical of SNe II models (Woosley $\&$ Weaver 1995). A consequence would be that a large fraction - over $10 \%$ - of the light nuclei $(\mathrm{O}, \mathrm{Mg}$ and so on) would also come from the same source. The isotopic variations that might be expected from such a contribution have not been observed. One must note that, if the SNe II debris were thoroughly mixed in the protosolar cloud, then there would be no direct observational test as the isotopic 'anomalies' would be homogenised; the solar abundances would then not be what we believe or assume them to be - that is, a well mixed average sample of the ISM at the time of the Sun's formation. The remarkable discovery of a large excess of pure ${ }^{16} \mathrm{O}(\sim 4 \%)$ in refractory CAIs (Clayton, Grossman, \& Mayeda 1973; Clayton, Mayeda, \& Onuma 1976) stimulated much of the search for shortlived nuclei. However, recently these oxygen anomalies have been attributed to mass-independent isotopic effects (Thiemens \& Heidenreich 1983) and not related to nuclear processes (Thiemens 1999). This alternative possibility has also been considered by Clayton (2002). A demonstration that the 'mass independent' isotopic fractionation can actually be responsible for the effects found in condensed phases has, however, not been made.

An objection to the supernova trigger hypothesis can be advanced on the basis of the increased knowledge of star formation in the Galaxy. There is a clear distinction between the mechanisms controlling the coalescence of isolated cores or globules and those at the base of clustered star formation. Only in the latter case are massive star neighbours present which may play a role in a sequential process of star formation. In the scenario of cluster formation, events of triggering do appear to be present (Kothes, Uyaniker, \& Pineault 2001; Palla \& Stahler 2000; Zinnecker 2002), induced by SN ejecta or by massive-star winds from $\mathrm{OB}$ associations. The distance from a possible triggering $\mathrm{SN}$ to the proto-sun can be roughly estimated assuming spherical symmetry. The mixing ratio needed to obtain the ESS abundances of short-lived species from $\mathrm{SN}$ ejecta of the same nuclei would require a distance of a few parsec (see Cameron et al. 1995). However, further consideration of SN remnant evolution is needed. Recent observational evidence shows that triggering occurs when the shock fronts have slowed velocities down to a few $\mathrm{km} \mathrm{s}^{-1}$ or less (Preibisch \& Zinnecker 1999; Stanke, McCaughrean, \& Zinnecker 2002). Such slow motions may require that the expanding $\mathrm{SN}$ shells have formed shocks within the ISM through their adiabatic and subsequently snowplow phases, travelling a distance perhaps of the order of $50 \mathrm{pc}$ (see, for example, Scheffler \& Elsässer 1988, Chapter 6). This is much larger than the value (2.5$10 \mathrm{pc}$ ) required by the Cameron et al. (1995) model. A SN trigger like the one originally suggested by Cameron \& 
Truran (1977) would appear to be a very rare event. Certainly there is a high rate of star formation in OB associations. If, instead, the sun formed alone, or in an isolated binary system as is often the rule for low-mass cloud cores, then a nearby supernova is not expected to be present at all.

The model of AGB contamination also requires a close encounter with an AGB star. This is hard to justify on statistical grounds (Kastner \& Myers 1994) and would require the simultaneous occurrence of two phenomena not physically associated (an isolated low-mass cloudcore collapse and a nearby planetary nebula or mass losing AGB star). However, as argued in the previous section, some ESS radioactivities require a close encounter with material from a stellar source of some type. Indications of the nature of such an occurrence and the efficiency with which debris would be trapped in a cloud core can be obtained from theoretical models of stellar nucleosynthesis for different masses and through simulations of the hydrodynamic interactions between a stellar shock and a cloud core (see Vanhala \& Boss 2000, 2002, and references therein). This can be done without fully justifying the statistical relevance of such a model. We will follow this approach. In any case, for any model requiring a nearby polluting source, there are a number of unanswered problems.

\section{The Nearby Stellar Source: a Reappraisal}

In the case of prompt contamination of the local ISM at $t=0$, whatever the source, a result analogous to equation (1) applies. At $t=\Delta_{1}$, (Wasserburg et al. 1998):

$$
\alpha_{\mathrm{R}, \mathrm{S}}^{\Delta_{1}}=\left(\frac{N_{\mathrm{R}}}{N_{\mathrm{S}}}\right)_{\Delta_{1}} \simeq \frac{q_{\mathrm{S}, \mathrm{w}}}{q_{0}} \times\left(\frac{N_{\mathrm{R}}}{N_{\mathrm{S}}}\right)_{\mathrm{w}} f_{0} \times e^{-\frac{\Delta_{1}}{\bar{\tau}_{\mathrm{R}}}}
$$

where $\alpha_{\mathrm{R}, \mathrm{S}}^{\Delta_{1}}$ is the abundance ratio (radioactive nucleus to stable reference nucleus) established in the solar nebula at $t=\Delta_{1}, q_{\mathrm{S}, \mathrm{w}} / q_{0}$ is the enrichment factor of the stable nucleus $S$ in the stellar wind ejected by the star relative to the ISM. $\left(N_{\mathrm{R}} / N_{\mathrm{S}}\right)_{\mathrm{w}}$ is the abundance ratio (radioactive to stable) in the wind, $f_{0}$ is the mixing ratio for $t=0$ and $\bar{\tau}_{\mathrm{R}}$ is the lifetime of nuclide R. Note that we use here the net abundances in the envelope of the contaminating source at the time of ejection, which is different from in our previous works. In the earlier works for an AGB source we used the ratio in the $\mathrm{He}$ shell and a dilution factor for mixing with the envelope. Here we have calculated the values in the envelope directly. Abundances measured in planetary differentiates that formed later, at $t=\Delta_{2}$ (as is the case for $\mathrm{Pd}$ ), can be obtained by applying the further exponential decay factor to the radioactive nuclei of interest. Absolute dating of both ESS samples and of planetary differentiates yield values for $\Delta_{2}$ of the order of few Myr. This is in accord with other arguments that require a relatively short but significant time delay for protoplanet formation. It is also possible that values of $\Delta_{2}$ are slightly different for different samples; this also means that, for example, the time delay to be considered for ${ }^{107} \mathrm{Pd}$ may be slightly but significantly different from the one related to ${ }^{60} \mathrm{Fe}$ measurements.

\subsection{Short-Lived Nuclei from a 'Local' Supernova}

The issues to be discussed here are the questions of which short-lived nuclei in the ESS might have been supplied by a 'local' triggering supernova and whether there are diagnostic characteristics that would identify SN contributions. We will not discuss the $r$-process contributions because of the need for diverse $r$-sources and also because of the questions relating to $\mathrm{SNe}$ II as sources of heavy $r$-nuclei as discussed earlier. Our focus will be on the lighter nuclides produced by SNe II following available models and recognising that $\mathrm{SNe}$ II are known to produce ${ }^{56} \mathrm{Ni}$. In an earlier report (Wasserburg et al. 1998), following the work of Timmes et al. (1995), we further investigated the problem of whether ${ }^{26} \mathrm{Al},{ }^{41} \mathrm{Ca},{ }^{60} \mathrm{Fe}$ and ${ }^{53} \mathrm{Mn}$ could be adequately provided by a SN II event, using the models of Woosley \& Weaver (1995) as a basis. As recognised by Timmes et al. (1995), the average number ratio of $\left({ }^{26} \mathrm{Fe} /{ }^{60} \mathrm{Fe}\right)_{\mathrm{w}}$ in the $\mathrm{SN}$ ejecta of these models is about 8.6, from a wide range of stellar models, the only clear exception being for $M \simeq 13 \mathrm{M}_{\odot}$. It was found that to match the $\left({ }^{26} \mathrm{Al} /{ }^{27} \mathrm{Al}\right)_{\Delta_{1}}$ ratio of $\sim 5 \times 10^{-5}$, a mixing ratio of $\sim 5 \times 10^{-4}$ was required and that this source yielded abundant $\left({ }^{41} \mathrm{Ca} /{ }^{40} \mathrm{Ca}\right)_{\Delta_{1}}$ and $\left({ }^{60} \mathrm{Fe} /{ }^{56} \mathrm{Fe}\right)_{\Delta_{1}}$ of about $10^{-6}$. In addition, the inferred relative abundance of ${ }^{53} \mathrm{Mn}$ implies that $\left({ }^{53} \mathrm{Mn} /{ }^{55} \mathrm{Mn}\right)_{\Delta_{1}}$ is about $10^{-3}$. This is far above the observed value shown in Table 1. A SN II model would also require that about $10 \%$ of all ${ }^{16} \mathrm{O}$ in the protosolar cloud, as well as large contributions to other major isotopes, was from the single SN II event. It was proposed that the $\left({ }^{26} \mathrm{Al} /{ }^{60} \mathrm{Fe}\right)$ ratio be used as a test of the SN II model.

A new generation of SN II nucleosynthesis models were computed by Rauscher et al. (2002) for a wide range of masses. An up-to-date set of reaction rates (both experimental and theoretical) was used, in conjunction with upgrades in the evolutionary code. These calculations did not include a parametric $r$-process model but treated neutron capture reactions during the intermediate and advanced evolutionary stages. As a result, several neutron capture nuclei are produced. In the Rauscher et al. (2002) paper, Tables 8 and 9 (published electronically) give the yields of both radioactive and stable nuclei. Using these results and the published ejected masses, we have calculated the abundances to be found in a protosolar cloud with $\left({ }^{26} \mathrm{Al} /{ }^{27} \mathrm{Al}\right)_{\Delta_{1}}$ of $5 \times 10^{-5}$ and $\left({ }^{41} \mathrm{Ca} /{ }^{40} \mathrm{Ca}\right)_{\Delta_{1}}$ of $1.5 \times 10^{-8}$. Matching these two values gives the corresponding mixing ratio $f_{0}$ and time interval $\Delta_{1}$ for initial star masses of $15 \mathrm{M}_{\odot}$ (as shown in Table 2, herein) and $25 \mathrm{M}_{\odot}$ (see Table 3 ). It can be seen that most short-lived nuclei are produced abundantly, with the only exception being ${ }^{107} \mathrm{Pd}$, which is somewhat low. It is clear however that this is not a critical matter. Note that we have not chosen $\Delta_{1}=0$ but found the values of $f_{0}$ and $\Delta_{1}$ consistent with the assumed ${ }^{26} \mathrm{Al}$ and ${ }^{41} \mathrm{Ca}$ values. The principal conclusion is that if ${ }^{26} \mathrm{Al}$ and ${ }^{41} \mathrm{Ca}$ are from a SN 
Table 2. Short-Lived Nuclei in the ESS from a $15 \mathrm{M}_{\odot} \mathrm{SN}$ (Data from Rauscher et al. 2002)

$M=15 \mathrm{M}_{\odot}, f_{0}=3.05 \times 10^{-4}, \Delta_{1}=1.09 \mathrm{Myr}$

\begin{tabular}{llrll}
\hline Rad. & Ref. & \multicolumn{1}{c}{$q_{\mathrm{S}}$} & $\left(N_{\mathrm{R}} / N_{\mathrm{S}}\right)_{\mathrm{w}}$ & $\left(N_{\mathrm{R}} / N_{\mathrm{S}}\right)_{\Delta_{1}}$ \\
\hline${ }^{26} \mathrm{Al}$ & ${ }^{27} \mathrm{Al}$ & 80.7 & $5.7 \times 10^{-3}$ & $5.0 \times 10^{-5}$ \\
${ }^{41} \mathrm{Ca}$ & ${ }^{40} \mathrm{Ca}$ & 4.7 & $1.5 \times 10^{-2}$ & $1.5 \times 10^{-8}$ \\
${ }^{53} \mathrm{Mn}$ & ${ }^{55} \mathrm{Mn}$ & 95.6 & 0.15 & $3.5 \times 10^{-3}$ \\
${ }^{107} \mathrm{Pd}$ & ${ }^{108} \mathrm{Pd}$ & 1.2 & $3.1 \times 10^{-2}$ & $1.1 \times 10^{-5}$ \\
${ }^{60} \mathrm{Fe}$ & ${ }^{56} \mathrm{Fe}$ & 107.78 & $2.4 \times 10^{-3}$ & $4.7 \times 10^{-5}$ \\
\hline
\end{tabular}

Table 3. Short-Lived Nuclei in the ESS from a $25 \mathrm{M}_{\odot} \mathrm{SN}$ (Data from Rauscher et al. 2002)

$M=25 \mathrm{M}_{\odot}, f_{0}=1.33 \times 10^{-4}, \Delta_{1}=1.26 \mathrm{Myr}$

\begin{tabular}{llrll}
\hline Rad. & Ref. & \multicolumn{1}{c}{$q_{\mathrm{S}}$} & $\left(N_{\mathrm{R}} / N_{\mathrm{S}}\right)_{\mathrm{w}}$ & $\left(N_{\mathrm{R}} / N_{\mathrm{S}}\right)_{\Delta_{1}}$ \\
\hline${ }^{26} \mathrm{Al}$ & ${ }^{27} \mathrm{Al}$ & 380.2 & $3.3 \times 10^{-3}$ & $5.0 \times 10^{-5}$ \\
${ }^{41} \mathrm{Ca}$ & ${ }^{40} \mathrm{Ca}$ & 286.5 & $1.8 \times 10^{-3}$ & $1.5 \times 10^{-8}$ \\
${ }^{53} \mathrm{Mn}$ & ${ }^{55} \mathrm{Mn}$ & 174.5 & 0.16 & $3.0 \times 10^{-3}$ \\
${ }^{107} \mathrm{Pd}$ & ${ }^{108} \mathrm{Pd}$ & 2.7 & $4.7 \times 10^{2}$ & $1.7 \times 10^{-5}$ \\
${ }^{60} \mathrm{Fe}$ & ${ }^{56} \mathrm{Fe}$ & 110.6 & $1.1 \times 10^{-3}$ & $9.0 \times 10^{-6}$ \\
\hline
\end{tabular}

II source, then the ratio $\left({ }^{60} \mathrm{Fe} /{ }^{56} \mathrm{Fe}\right)_{\Delta_{1}}$ must be very high (in the range $\left.1-5 \times 10^{-5}\right)$ and $\left({ }^{53} \mathrm{Mn} /{ }^{55} \mathrm{Mn}\right)_{\Delta_{1}}$ must be extremely high. The new results are essentially consistent with the values calculated from the earlier models, but with some major differences; the extent to which this abundance pattern is diagnostic for $\mathrm{SNe}$ II will be discussed below.

The greatest discrepancy with the ESS data is the high value of ${ }^{53} \mathrm{Mn} /{ }^{55} \mathrm{Mn}$. Comparison of the values in Tables 2 and 3 with those in Table 1 shows that there is a difference of a factor of $10^{2}$. Discussion of ${ }^{60} \mathrm{Fe}$ is complex, because of the uncertainty in the ratio $\left({ }^{60} \mathrm{Fe} /{ }^{56} \mathrm{Fe}\right)_{\mathrm{ESS}}$. However, in both Tables 2 and 3 , the value of $\left({ }^{60} \mathrm{Fe} /{ }^{56} \mathrm{Fe}\right)_{\Delta_{1}}$ for a SN II model is high by a factor of 10 with respect to the present upper limit obtained from ESS measurements.

Note that Rauscher et al. (2002) give an average ${ }^{26} \mathrm{Al} /{ }^{60} \mathrm{Fe}$ ratio of approximately 1.5 . This is a factor of about 5.7 less than previously found by Woosley \& Weaver (1995). This ratio is now in direct conflict with the upper limits set by $\gamma$-ray observations. Note that such a value would considerably increase the importance of ${ }^{60} \mathrm{Fe}$ as a heat source for melting planetesimals in the ESS, compared with previous estimates (Wasserburg et al. 1998; see also Mostefaoui et al. 2003). Recently, new models have been presented by Chieffi \& Limongi (2003) in which their ${ }^{26} \mathrm{Al} /{ }^{60} \mathrm{Fe}$ ratios are more similar to those of Woosley \& Weaver (1995). In summary, for a SN II source and using the Rauscher et al. (2002) models, we find the following results:

(i) The mixing ratio is roughly compatible with the amount of mass necessary to slow down the ejecta to local velocities $\left(4 \times 10^{4} \mathrm{M}_{\odot}\right.$ of ISM for $\sim 10 \mathrm{M}_{\odot}$ of ejecta). The time scale becomes $\sim 10^{6} \mathrm{yr}$ if one matches the ESS values of ${ }^{26} \mathrm{Al} /{ }^{27} \mathrm{Al}$ and ${ }^{41} \mathrm{Ca} /{ }^{40} \mathrm{Ca}$. (ii) However, the ${ }^{53} \mathrm{Mn} /{ }^{55} \mathrm{Mn}$ is above any of the extensive determinations by Lugmair \& Shukolyukov (1998).

(iii) Further, it is evident that ${ }^{60} \mathrm{Fe} /{ }^{56} \mathrm{Fe}$ is also far above existing data.

We note that the ${ }^{53} \mathrm{Mn}$ overproduction has led Meyer $\&$ Clayton (2000) to suggest that the source was a peculiar massive star that ejected only the external envelope rich in ${ }^{26} \mathrm{Al}$ and ${ }^{60} \mathrm{Fe}$ and not the innermost region rich in ${ }^{53} \mathrm{Mn}$. This ad hoc model is difficult to evaluate at the present time without further predictions based on the same idea. However, as ${ }^{60} \mathrm{Fe}$ is produced in layers external to the innermost regions producing ${ }^{53} \mathrm{Mn}$, increasing the mass cut (to reduce ${ }^{53} \mathrm{Mn}$ ) would leave, at least, the ${ }^{60} \mathrm{Fe}$ problem unresolved. An important source of concern has been added by Sahijpal \& Soni (2003), who showed that all massive star scenarios (SN II, SN Ib/c, non-exploding Wolf-Rayet stars) would provide large contributions of intermediate mass elements to the protosolar cloud. This was discussed earlier by Wasserburg et al. (1998) for SN II sources. All these difficulties should be considered together: while each of them (such as the increased problems in the ${ }^{26} \mathrm{Al} /{ }^{60} \mathrm{Fe}$ ratio deriving from the work of Rauscher et al. 2002) might be individually ascribed to uncertainties in cross sections or model details, we believe that the existence of several correlated reasons of concern (with the abundance of ${ }^{53} \mathrm{Mn}$ being the dominant problem) makes the SN trigger model rather unlikely amongst the available models and data. As indicated above, there is at present no evidence of material from such a supernova, unless of course it was thoroughly mixed. We further note that the overwhelming abundance of presolar grains found in meteorites is from AGB stars and not from SNe II.

\subsection{Short-Lived Nuclei from an AGB Star}

The alternative scenario of an AGB star polluting the Early Solar Nebula was first explored quantitatively by Wasserburg et al. (1994). They concluded that AGB scenarios suitably able to accomplish this task could be found among stellar models with low amounts of neutron captures. This corresponds to the absence or very small concentration of the extra ${ }^{13} \mathrm{C}$ neutron source that is assumed to be present in AGB stars in order to explain the bulk of $s$-process production for average solar system abundances (Gallino, Raiteri, \& Busso 1993). This ${ }^{13} \mathrm{C}$ reservoir is known (from observations) to be variable, being different in different classes of AGB stars. This results in a large spread of neutron exposures, corresponding to ${ }^{13} \mathrm{C}$ amounts in the pocket ranging from zero to a few times $10^{-6} \mathrm{M}_{\odot}$ (Busso et al. 2001; Abia et al. 2002). The ${ }^{13} \mathrm{C}$ pocket drives $s$-processing under radiative conditions (Straniero et al. 1997; Gallino et al. 1998) in the time interval between two successive thermal pulses. In the absence of this neutron source, the only neutron captures experienced in the star are those occurring during the convective pulses, due to the ${ }^{22} \mathrm{Ne}(\alpha, \mathrm{n})^{25} \mathrm{Mg}$ reaction. This last reaction naturally derives from transformation of ${ }^{14} \mathrm{~N}$ accumulated by $\mathrm{H}$ burning, followed by 


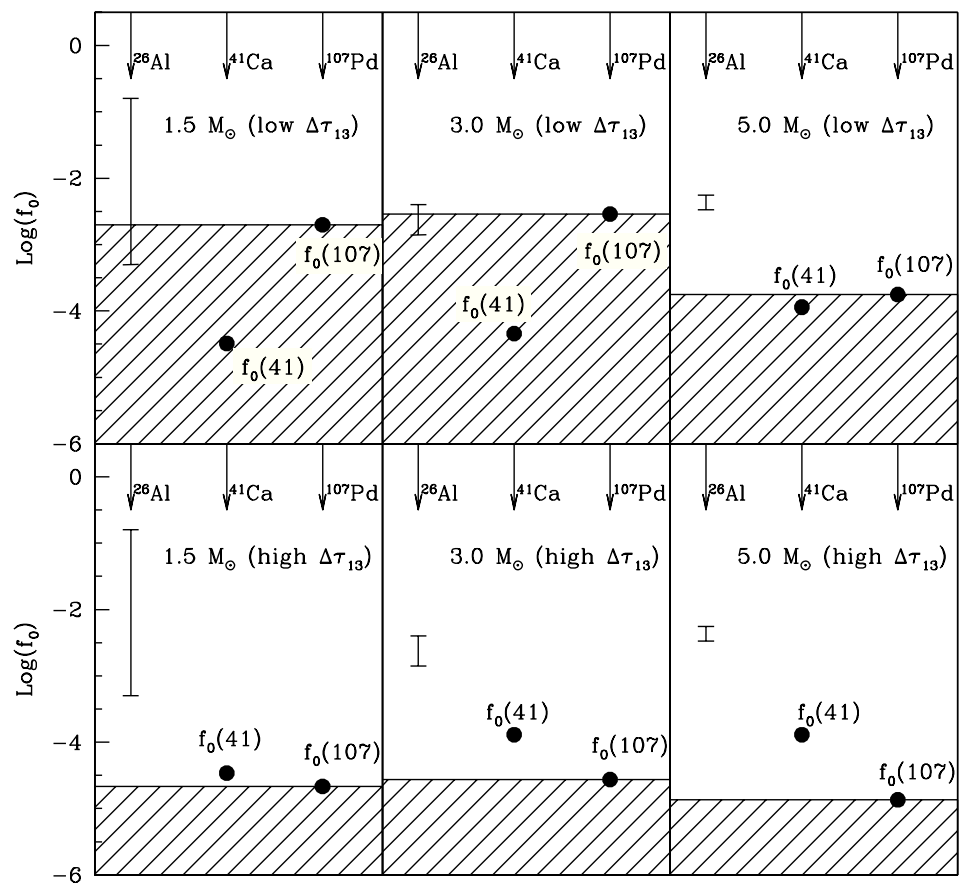

Figure 1 Mixing ratios between the processed envelope material of a solar metallicity AGB star and the ISM from which the solar system formed, for the short-lived nuclei ${ }^{26} \mathrm{Al},{ }^{41} \mathrm{Ca}$ and ${ }^{107} \mathrm{Pd}$. The value shown for each isotope is that which is necessary to account for its ESS measurement at $\Delta=0$ for each adopted AGB model. In order to obtain a global solution for the three nuclei together, only cases where the mixing ratio derived for ${ }^{107} \mathrm{Pd}, f_{0}(107)$, is larger than the other two (and hence these must fall in the shaded area below ${ }^{107} \mathrm{Pd}$ ) can be considered, because then radioactive decay can bring the abundances into agreement at a time $\Delta>0$. Due to this, only the first two panels (in bold) are acceptable. Note that for $1.5 \mathrm{M}_{\odot}$ models, the large range given for ${ }^{26} \mathrm{Al}$ is between no cool bottom processes to reasonable contributions from them (Nollett et al. 2003). No cool bottom process was considered in the $3 \mathrm{M}_{\odot}$ models. Hot bottom burning was included in the models for the $5 \mathrm{M}_{\odot}$ star.

the $\alpha$ chain ${ }^{14} \mathrm{~N}(\alpha, \gamma){ }^{18} \mathrm{~F}\left(\beta^{+} \nu\right){ }^{18} \mathrm{O}(\alpha, \gamma){ }^{22} \mathrm{Ne}$. Thus there is plenty of ${ }^{22} \mathrm{Ne}$. The ${ }^{22} \mathrm{Ne}$ neutron source is only marginally activated in low mass stars $\left(M \leq 3 \mathrm{M}_{\odot}\right)$ due to the low temperature in the pulses $\left(T \leq 3 \times 10^{8} \mathrm{~K}\right)$, but is more significant for higher masses (intermediate mass stars, $3 \mathrm{M}_{\odot} \leq M \leq 8 \mathrm{M}_{\odot}$ ), where the maximum temperature in the bottom of the thermal pulse may reach $3.5 \times 10^{8} \mathrm{~K}$.

A new set of calculations was made for this report using updated cross sections, both considering the neutron flux from a typical ${ }^{13} \mathrm{C}$ pocket, and excluding it. Calculations were done for stellar models of $1.5,3$ and $5 \mathrm{M}_{\odot}$ at various metallicities. When a ${ }^{13} \mathrm{C}$ pocket was considered, the standard (ST) choices presented by Gallino et al. (1998) and Busso et al. (2001) were adopted, corresponding to the burning of $4 \times 10^{-6} \mathrm{M}_{\odot}$ of ${ }^{13} \mathrm{C}$ per cycle in low mass stars and of $4 \times 10^{-7} \mathrm{M}_{\odot}$ in intermediate mass stars. These cases will be referred to as having 'high $\Delta \tau_{13}$ ', where $\Delta \tau_{13}$ is the neutron exposure produced in the ${ }^{13} \mathrm{C}$ pocket ( $\Delta \tau_{13}=\int N_{n} v_{t h} d t$, where $N_{n}$ is the neutron density and $v_{t h}$ is the thermal velocity). Models were run through the full evolution of the star and the final values of the abundance ratios for short-lived isotopes in the envelope (such as ${ }^{41} \mathrm{Ca} /{ }^{40} \mathrm{Ca}$ ) were calculated, taking into account dredgeup, temperature-dependent decay in the various stellar layers and mass loss. The mass loss was parametrised through the Reimers (1975) formula: choices of this free parameter are discussed elsewhere (Gallino et al. 1998; Busso et al. 2001; Abia et al. 2002). The results presented here give essentially the same He shell composition as in Busso et al. (1999), but here we directly estimate envelope abundances at the end of the AGB evolution. This permits straightforward derivation of the mixing ratios for the various nuclei in the ISM.

Our estimated error bars for the ratios in the envelope do not exceed a factor of $\sim 2$, with the exception of ${ }^{26} \mathrm{Al}$, for which the uncertainty is still large. For this nucleus the range of values given refers to the maximum interval covered by present models. In our calculations, the production of ${ }^{26} \mathrm{Al}$ computed from the $\mathrm{H}$ and $\mathrm{He}$ shell is used, with the addition of recent estimates of its synthesis in the envelope, either through hot bottom burning in intermediate mass stars or through cool bottom processes in low mass stars. The range of the ensuing possibilities for the contribution to ${ }^{26} \mathrm{Al}$ is especially large for low mass stars, spanning the range from pure (limited) production in the $\mathrm{H}$ and $\mathrm{He}$ shells, to much higher values expected from application of cool bottom process models with high efficiency (Nollett et al. 2003). The range is more restricted for a $3 \mathrm{M}_{\odot}$ star, shown in Figure 1, because no cool bottom processes have been considered. So far, no models of cool bottom processes have been attempted for masses in the $2-3 \mathrm{M}_{\odot}$ range. If analogy to the red giant branch 
(RGB) applies (which is not certain), then the efficiency of cool bottom process should decrease with increasing stellar mass. The range formally spanned by ${ }^{26} \mathrm{Al}$ yields is even smaller for a $5 \mathrm{M}_{\odot}$ star, as recent models for hot bottom burning converge toward rather well-defined production factors for it in the envelope (Lattanzio \& Forestini 1999; Lugaro et al. 2001).

The possibility of obtaining solutions for short-lived nuclei in the ESS from AGB models is illustrated in Figure 1 , for cases including or excluding the formation of a ${ }^{13} \mathrm{C}$ source. For simplicity, we calculate the mixing ratio $f_{0}$ needed to produce the corresponding individual ESS abundance ratios of each of the three nuclides for $\Delta_{1}=0$ (that is, for no time delay $\Delta$ between the nucleosynthesis event and the formation of first condensates). The above treatment, although oversimplified, is nevertheless useful. In it we can immediately recognise that a necessary condition for a model to provide a unique solution for ${ }^{107} \mathrm{Pd}$, ${ }^{41} \mathrm{Ca}$ and ${ }^{26} \mathrm{Al}$ is that the value of $f_{0}$ derived above for ${ }^{107} \mathrm{Pd}$ must be much greater than for the other two. In particular, it must be far larger than for ${ }^{41} \mathrm{Ca}$. Note that ${ }^{107} \mathrm{Pd}$ has a longer meanlife than ${ }^{26} \mathrm{Al}$ (by a factor of 10 ) and ${ }^{41} \mathrm{Ca}$ (by a factor of 60). Hence, a larger $f_{0}$ deduced for ${ }^{107} \mathrm{Pd}$ (at $\Delta_{1}=0$ ) would allow the other two values to be brought into agreement by assuming free decay for a time interval $\Delta_{1}$. For $\Delta_{1}$ of the order of $1 \mathrm{Myr}$, the result would be to greatly reduce ${ }^{41} \mathrm{Ca}$ and even decrease ${ }^{26} \mathrm{Al}$, without affecting ${ }^{107} \mathrm{Pd}$. On the contrary, any case corresponding to a value $f_{0}$ for ${ }^{107} \mathrm{Pd}$ equal to or lower than those deduced from the others at $t=0$ would inevitably fail to yield a solution for any $\Delta_{1}>0$. With these basic rules in mind we see that the only acceptable cases are in the range of low AGB stellar masses with low $s$-process efficiency, where ${ }^{107} \mathrm{Pd}$ provides the required values of $f_{0}$ and then a solution is possible that permits $\Delta_{1} \geq 0$. We note instead that the $5.0 \mathrm{M}_{\odot}$ model with low neutron exposure requires almost immediate injection of material in order to provide ${ }^{41} \mathrm{Ca}$ and would fail to match the ${ }^{26} \mathrm{Al}$ data.

We stated that the condition on $f_{0}$ is a necessary one. For it also to be sufficient depends on the possibility of finding a specific solution with reasonable values for $f_{0}, \Delta_{1}$ and $\Delta_{2}$ (see eq. (2) and the subsequent discussion). When this is possible, one can further try a direct comparison of the species ${ }^{107} \mathrm{Pd},{ }^{41} \mathrm{Ca}$ and ${ }^{26} \mathrm{Al}$ with the observed abundances of ${ }^{60} \mathrm{Fe}$. A self-consistent solution would require a fixed mass for the AGB star and also the same values of $f_{0}, \Delta_{1}$ and $\Delta_{2}$ for all four species (although see comment immediately before Section 4.1 ). The ${ }^{60} \mathrm{Fe}$ is especially critical, as it does not depend on the magnitude of the ${ }^{13} \mathrm{C}$ pocket which is a free parameter of $s$-processing in AGB stellar models.

For the $1.5 \mathrm{M}_{\odot}$ model, a self-consistent solution is found for $f_{0} \sim 5 \times 10^{-3}, \Delta_{1} \sim 0.8 \mathrm{Myr}, \Delta_{2} \sim 7 \mathrm{Myr}$. This solution is presented in Table 4 . In the second panel of the table we also show the ensuing abundance ratios for a number of short-lived isotopes produced in the AGB model, whose presence and/or quantitative abundance in the ESS still needs a confirmation; we leave these data as
Table 4. Short-lived Nuclei from a Low Mass Star with Cool Bottom Processes

$$
\begin{gathered}
M=1.5 \mathrm{M}_{\odot}, Z=0.02, f_{0}=5.1 \times 10^{-3}, \Delta_{1}=0.76 \mathrm{Myr}, \\
\Delta_{2}=7 \mathrm{Myr}
\end{gathered}
$$

\begin{tabular}{llllll}
\hline Rad. & Ref. & $q_{\mathrm{S}}$ & $\left(N_{\mathrm{R}} / N_{\mathrm{S}}\right)_{\mathrm{W}}$ & $\left(N_{\mathrm{R}} / N_{\mathrm{S}}\right)_{\Delta_{1}}$ & $\left(N_{\mathrm{R}} / N_{\mathrm{S}}\right)_{\Delta_{2}}$ \\
\hline${ }^{26} \mathrm{Al}(*)$ & ${ }^{27} \mathrm{Al}$ & 1.02 & $2.0 \times 10^{-2}$ & $5.0 \times 10^{-5}$ & $6.4 \times 10^{-8}$ \\
${ }^{41} \mathrm{Ca}$ & ${ }^{40} \mathrm{Ca}$ & 0.99 & $4.7 \times 10^{-4}$ & $1.5 \times 10^{-8}$ & $\simeq 0$ \\
${ }^{60} \mathrm{Fe}$ & ${ }^{56} \mathrm{Fe}$ & 0.99 & $9.3 \times 10^{-6}$ & $3.3 \times 10^{-8}$ & $1.4 \times 10^{-9}$ \\
${ }^{107} \mathrm{Pd}$ & ${ }^{108} \mathrm{Pd}$ & 1.01 & $9.8 \times 10^{-3}$ & $4.5 \times 10^{-5}$ & $2.0 \times 10^{-5}$ \\
${ }^{36} \mathrm{Cl}$ & ${ }^{35} \mathrm{Cl}$ & 0.99 & $2.7 \times 10^{-3}$ & $2.4 \times 10^{-6}$ & $\simeq 0$ \\
${ }^{93} \mathrm{Zr}$ & ${ }^{92} \mathrm{Zr}$ & 1.01 & $1.1 \times 10^{-2}$ & $4.0 \times 10^{-5}$ & $1.7 \times 10^{-6}$ \\
${ }^{99} \mathrm{Tc}$ & ${ }^{100} \mathrm{Ru}$ & 1.02 & $7.1 \times 10^{-3}$ & $3.2 \times 10^{-6}$ & $\simeq 0$ \\
${ }^{135} \mathrm{Cs}$ & ${ }^{133} \mathrm{Cs}$ & 0.99 & $2.3 \times 10^{-2}$ & $1.1 \times 10^{-4}$ & $2.2 \times 10^{-5}$ \\
${ }^{205} \mathrm{~Pb}$ & ${ }^{204} \mathrm{~Pb}$ & 1.07 & $1.3 \times 10^{-1}$ & $6.6 \times 10^{-4}$ & $4.8 \times 10^{-4}$ \\
\hline
\end{tabular}

(*) Estimate including cool bottom processes from Nollett et al. (2003).

predictions, waiting for a test by new measurements. In interpreting the data of Table 4, one has to consider the following:

(i) For ${ }^{205} \mathrm{~Pb}$, calculations have an intrinsic uncertainty related to the still incomplete knowledge of its electron captures as a function of temperature for the range $10^{6}$ $1.5 \times 10^{8} \mathrm{~K}$ (see Yokoi, Takahashi, \& Arnould 1985; Wasserburg et al. 1994). In view of the choices made in the calculations, the datum of Table 4, column 4, must be seen as an upper limit.

(ii) It is well known that ${ }^{99} \mathrm{Tc}$ is produced in AGB stars (Merrill 1952). While no direct evidence for this shortlived nucleus has so far been found in meteorites, there are now new data indicating isotopic effects in $\mathrm{Ru}$ (Chen, Papanastassiou, \& Wasserburg 2003).

The value in Table 4, when expressed at $\Delta_{1}=0$, corresponds to ${ }^{99} \mathrm{Tc} /{ }^{100} \mathrm{Ru} \approx 3.7 \times 10^{-5}$ (a similar value, $2.3 \times 10^{-5}$, was found by Wasserburg et al. 1994). This is not very sensitive to the presence of $a^{13} \mathrm{C}$ pocket if ${ }^{107} \mathrm{Pd}$ is matched, since the abundance ratio between ${ }^{99} \mathrm{Tc}$ and ${ }^{107} \mathrm{Pd}$ is almost independent of the neutron exposure. The detection of ${ }^{99} \mathrm{Tc}$ in meteoritic material would be very important. However, as noted by Chen et al. (2003), the chemical fractionation (for example, enrichment-depletion) pattern of Tc to be expected in meteoritic materials is completely unknown.

The solution shown in Table 4 would require ${ }^{26} \mathrm{Al} /{ }^{27} \mathrm{Al}$ $\sim 2 \times 10^{-2}$ in the envelope, which could be readily reached by cool bottom processing (Nollett et al. 2003).

For the $3 \mathrm{M}_{\odot}$ model, from Figure 1, one would find a discrepancy for ${ }^{26} \mathrm{Al}$. In order to obtain a self-consistent solution for this case, we would need to increase the ${ }^{26} \mathrm{Al}$ production by a factor of about 3 . This shows that good estimates are urgently needed of the efficiency of cool bottom processes for stellar masses above that of the only case $\left(1.5 \mathrm{M}_{\odot}\right)$ so far explored. Calculations of the concurrent effects on ${ }^{26} \mathrm{Al}$ synthesis in the range $2-3 \mathrm{M}_{\odot}$ should clarify whether or not this nucleus can be provided in sufficient amounts. We conclude that acceptable 
scenarios might have AGB sources in the mass range $1.5 \mathrm{M}_{\odot} \leq M \leq 3 \mathrm{M}_{\odot}$. These solutions do not extend to ${ }^{182} \mathrm{Hf}$, despite the fact that this nucleus receives small contributions from the $s$-process. As noted in previous works, the AGB production is in this case grossly insufficient. Issues regarding ${ }^{182} \mathrm{Hf}$ are as described in Wasserburg et al. (1996); its ESS inventory must be ascribed to the long term production of the heaviest $r$-process nuclei (with a lower cutoff at Ba, see Qian et al. 1998a).

\subsection{The ${ }^{60} \mathrm{Fe}$ Problem}

Concerning the more difficult case of ${ }^{60} \mathrm{Fe}$, it was recalled in the previous section that for $\mathrm{SNe}$ II ${ }^{60} \mathrm{Fe}$ is produced at exceedingly high abundances compared with ${ }^{26} \mathrm{Al}$. We now consider the question of whether it might be produced in suitable amounts in AGB stars. Our previous investigation (Wasserburg et al. 1994) showed that significant ${ }^{60} \mathrm{Fe}$ production takes place in the He shell of AGB stars. The physics of AGB stars appears to be rather well defined (see Busso et al. 1999). The nuclear reaction rates involved are provided by extensive and sophisticated laboratory studies at the appropriate energies (Bao et al. 2000). As a result, the model of an AGB source for shortlived nuclei in the ESS has very strict constraints. As the envelope of an AGB star is well mixed, its composition is fixed by the initial conditions (mass and metallicity) and the three dynamical variables: the degree of neutron exposure in the radiative zone (that is, the ${ }^{13} \mathrm{C}$ pocket), the mass loss and the degree of cool bottom processing. The list of nuclei that can be made and those that cannot be made (such as ${ }^{10} \mathrm{Be},{ }^{53} \mathrm{Mn}$ and ${ }^{129} \mathrm{I}$ ) is explicit both qualitatively and quantitatively; in particular, the nuclide ${ }^{60} \mathrm{Fe}$ is a required product of AGB stars. In general, the main uncertainty lies in the rule used for determining mass loss.

Synthesis of ${ }^{60} \mathrm{Fe}$ requires two neutron captures, first on stable ${ }^{58} \mathrm{Fe}$ and then on unstable ${ }^{59} \mathrm{Fe}(\bar{\tau}=65.4$ days $)$. Operation of the reaction branching here, through the neutron-rich channel feeding ${ }^{60} \mathrm{Fe}$, needs rather high neutron densities. These densities can be achieved only by the ${ }^{22} \mathrm{Ne}$ neutron source in the pulses, where the temperature is high enough. Hence, virtually no ${ }^{60} \mathrm{Fe}$ production occurs in the low-neutron density ${ }^{13} \mathrm{C}$-burning phase. Wasserburg et al. (1994) found a local ratio in the production zone for ${ }^{60} \mathrm{Fe} /{ }^{56} \mathrm{Fe}$ of $10^{-4}$ to $10^{-3}$. This provided full compatibility of an AGB source with estimates of the ESS inventory then available. Updated computations for a $1.5 \mathrm{M}_{\odot}$ star of solar metallicity (Busso et al. 1999) subsequently gave a ${ }^{60} \mathrm{Fe} /{ }^{56} \mathrm{Fe}$ ratio of $1.9 \times 10^{-4}$ for the He shell. We present here the more extended results of the models mentioned above, in which we have determined the evolution of the ${ }^{60} \mathrm{Fe} /{ }^{56} \mathrm{Fe}$ ratio in the envelope pulse after pulse. These ratios do not depend on the uncertain choices for the ${ }^{13} \mathrm{C}$ pocket formed in the radiative interpulse phases, as ${ }^{60} \mathrm{Fe}$ is entirely produced in the convective pulses.

The extent to which an AGB source could quantitatively provide sufficient ${ }^{60} \mathrm{Fe}$ for the ESS is at present a problem and may be the key to understanding the
Table 5. Abundance Ratios ${ }^{60} \mathrm{Fe} /{ }^{56} \mathrm{Fe}$ and Overabundance $q_{56}$ in the Stellar wind (w), at the End of the AGB Evolution for the Models Shown in Figure 2

\begin{tabular}{llll}
\hline Mass & Metallicity & $\left(N_{60} / N_{56}\right)_{\mathrm{w}}$ & $q_{56, \mathrm{w}}$ \\
\hline $1.5 \mathrm{M}_{\odot}$ & 0.006 & $5.4 \times 10^{-5}$ & 0.29 \\
& 0.01 & $1.7 \times 10^{-5}$ & 0.50 \\
& 0.02 & $9.3 \times 10^{-6}$ & 0.99 \\
$3.0 \mathrm{M}_{\odot}$ & 0.006 & $1.6 \times 10^{-3}$ & 0.29 \\
& 0.01 & $5.9 \times 10^{-5}$ & 0.50 \\
& 0.02 & $3.4 \times 10^{-5}$ & 0.99 \\
$5.0 \mathrm{M}_{\odot}$ & 0.006 & $4.5 \times 10^{-3}$ & 0.29 \\
& 0.01 & $4.0 \times 10^{-3}$ & 0.49 \\
& 0.02 & $3.1 \times 10^{-3}$ & 0.98 \\
\hline
\end{tabular}

nature of the contaminating stellar source. A major uncertainty in the ${ }^{60} \mathrm{Fe}$ production lies in the neutron capture cross sections of both ${ }^{59} \mathrm{Fe}$ and ${ }^{60} \mathrm{Fe}$ which are based on theoretical estimates only. The Maxwellianaveraged cross section of ${ }^{60} \mathrm{Fe}$ at $30 \mathrm{keV}$ is rather low: $\langle\sigma\rangle(30 \mathrm{keV})\left({ }^{60} \mathrm{Fe}\right)=3.65 \mathrm{mbarn}$, according to Woosley et al. (1978). Consequently, only a small fraction of the ${ }^{60} \mathrm{Fe}$ produced can undergo further neutron captures. On the other hand, ${ }^{60} \mathrm{Fe}$ synthesis directly depends on the cross section of ${ }^{59} \mathrm{Fe}$, whose value at $30 \mathrm{keV}$, as estimated by Woosley et al. (1978), is 12.3 mbarn. More recent calculations, based on a different theoretical approach, have been performed by Rauscher \& Thielemann (2000) who obtain $\langle\sigma\rangle(30 \mathrm{keV})\left({ }^{59} \mathrm{Fe}\right)=22.5 \mathrm{mbarn}$. We adopt estimates here from Woosley et al. (1978), but we verified that, by using instead the Rauscher \& Thielemann (2000) cross sections for both ${ }^{59} \mathrm{Fe}$ and ${ }^{60} \mathrm{Fe}$, our production of ${ }^{60} \mathrm{Fe}$ (Table 5) would be increased by factors of about 1.8 for all the $1.5 \mathrm{M}_{\odot}$ and $3 \mathrm{M}_{\odot}$ models and about 1.2 for the $5 \mathrm{M}_{\odot}$ models. We may conclude that our predictions cannot change, due to variations in the neutron capture cross sections, by more than a factor of two. With this limit in mind, we have calculated the mixing factor $f_{0}$ necessary to achieve a given value of $\left(N_{60} / N_{56}\right)_{0}$ for the case of $\Delta_{1}=0$ for three stellar masses of $1.5,3.0$ and $5 \mathrm{M}_{\odot}$, each for three different metallicities $(Z=0.02,0.01$, and 0.006). The resulting mixing factors are shown in Figure 2 and the corresponding envelope abundances for ${ }^{60} \mathrm{Fe}$ and ${ }^{56} \mathrm{Fe}$ are shown in Table 5 . It can be seen that for $M=$ $1.5 \mathrm{M}_{\odot}$ and $Z=0.02$, a value of $f_{0}=10^{-2}$ is obtained for $\left(N_{60} / N_{56}\right)_{0}$ of $10^{-7}$. To achieve the higher value of $10^{-6}$ for $\left(N_{60} / N_{56}\right)_{0}$ (Mostefaoui et al. 2003) for this stellar mass, a high value of $f_{0}(\sim 0.1)$ would be required. For the case of a $3 \mathrm{M}_{\odot}$ star, values for $\left(N_{60} / N_{56}\right)_{0}$ of $3 \times 10^{-7}$ are readily achieved for $f_{0} \leq 10^{-2}$ at $Z=0.02$ and higher $\left(N_{60} / N_{56}\right)_{0}$ values can be obtained for $Z=0.006$. The $5 \mathrm{M}_{\odot}$ star can readily provide $\left(N_{60} / N_{56}\right)_{0}$ values up to $10^{-6}$ for $f_{0}<10^{-3}$. If we require the AGB source to have close to solar $Z$, then the value of $\left(N_{60} / N_{56}\right)_{\mathrm{ESS}}$ must be $\lesssim 3 \times 10^{-7}$ for a $3.0 \mathrm{M}_{\odot}$ star and $\lesssim 1 \times 10^{-7}$ for a $1.5 \mathrm{M}_{\odot}$ star. (We note that the global solution for the 


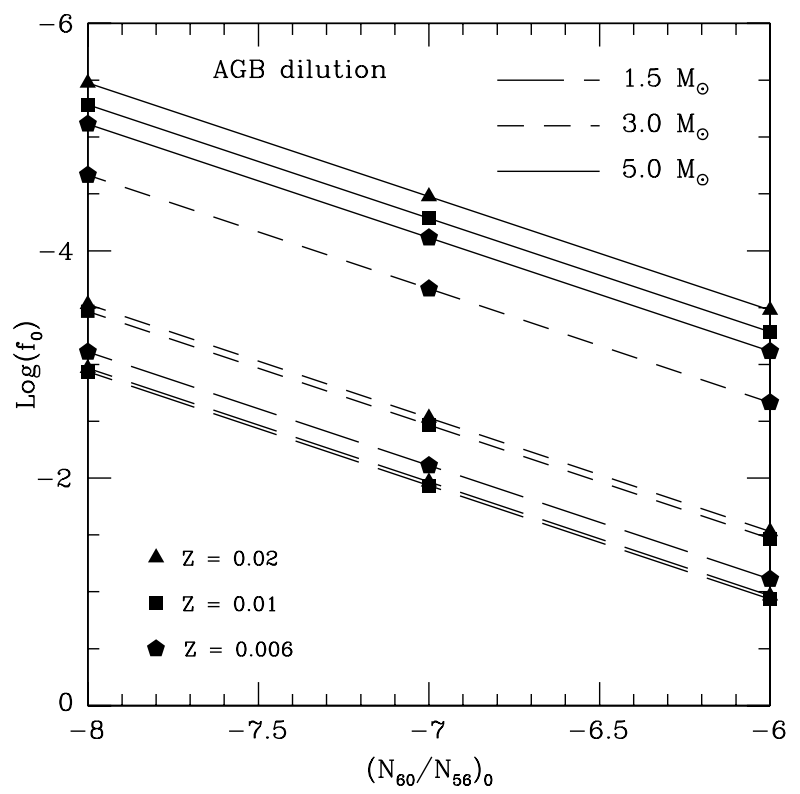

Figure 2 Mixing ratios $f_{0}$ between an AGB envelope material and local ISM needed to account for an ESS ${ }^{60} \mathrm{Fe} /{ }^{56} \mathrm{Fe}$ ratio in the wide range covered by measurement uncertainties. Models refer to different AGB masses and metallicities as indicated in the labels. The bottom three lines (long dash) are for the $1.5 \mathrm{M}_{\odot}$ case for different values of $Z$. The middle three lines (short dash) are for $3 \mathrm{M}_{\odot}$ and the unbroken top three lines are for $5 \mathrm{M}_{\odot}$.

$1.5 \mathrm{M}_{\odot}$ model, including ${ }^{26} \mathrm{Al},{ }^{41} \mathrm{Ca}$ and ${ }^{107} \mathrm{Pd}$, yields the lower value of $\left.\left({ }^{60} \mathrm{Fe} /{ }^{56} \mathrm{Fe}\right)\right)_{\Delta_{1}}=3 \times 10^{-8}$, see Table 4.) In general, considering the results for ${ }^{60} \mathrm{Fe}$ and the range of possible solutions for the other nuclei, it appears that an AGB source with $1.5 \mathrm{M}_{\odot} \lesssim M \lesssim 3 \mathrm{M}_{\odot}$ still offers plausible possibilities for explaining ${ }^{60} \mathrm{Fe}$ together with ${ }^{26} \mathrm{Al}$, ${ }^{41} \mathrm{Ca}$ and ${ }^{107} \mathrm{Pd}$.

Evidence for the presence of ${ }^{60} \mathrm{Fe}$ was first seen by Birck \& Lugmair (1988) who found variations in the abundances of $\mathrm{Ni}$ isotopes in CAIs from Allende with an excess of ${ }^{60} \mathrm{Ni}$. However, these effects were not correlated with $\mathrm{Fe} / \mathrm{Ni}$. If these variations were attributed to ${ }^{60} \mathrm{Fe}$, then this would correspond to $\left(N_{60} / N_{56}\right)_{\Delta_{1}} \approx(1.5 \pm 0.5) \times 10^{-6}$. However, it has been well known that there are general isotopic anomalies (variability in isotopic composition) both in CAIs and other ESS material. These effects are not obviously attributable to short-lived nuclei. The demonstration of isotopic shifts in the daughter nuclide must be correlated with the parent element abundance as discussed above. Evidence for the presence of ${ }^{60} \mathrm{Fe}$ was found in the planetary differentiates Chervony Kut, Juvinas and Caldera (Shukolyukov \& Lugmair, 1993a, 1993b, 1996). However, no clear correlation was found for ${ }^{60} \mathrm{Ni}$ excesses with $\mathrm{Fe}$ in these samples and the data are widely scattered. Nonetheless, there was some indication that ${ }^{60} \mathrm{Fe}$ was present after protoplanets formed $\left(\Delta_{2} \geq 0\right.$, see ref. 5 in Table 1). The initial abundance then is critically dependent on the values of $\left({ }^{60} \mathrm{Fe} /{ }^{56} \mathrm{Fe}\right)_{\Delta_{2}}$ as inferred from the data $\left(3.9 \times 10^{-9}\right.$ to $\left.4.3 \times 10^{-10}\right)$ and an estimate for the time scale $\Delta_{2}$. The most recent efforts dedicated to the search for ${ }^{60} \mathrm{Fe}$ occurred in studies of unequilibrated ordinary chondrites by measuring Ni deficient FeS phases. The critical reports are by Tachibana and Huss (2003a, 2003b) who found $\left({ }^{60} \mathrm{Fe} /{ }^{56} \mathrm{Fe}\right)_{\text {UOC }}$ ratios of $(1.08 \pm 0.23) \times 10^{-7}$ and $(1.73 \pm 0.53) \times 10^{-7}$ and by Mostefaoui et al. (2003) who found $\left({ }^{60} \mathrm{Fe} /{ }^{56} \mathrm{Fe}\right)_{\mathrm{UOC}}=7.5 \times 10^{-7}$. Both groups sought to establish correlations of ${ }^{60} \mathrm{Ni} /{ }^{61} \mathrm{Ni}$ with ${ }^{56} \mathrm{Fe} /{ }^{61} \mathrm{Ni}$. The evidence in the above three reports appears quite convincing for the presence of ${ }^{60} \mathrm{Fe}$ at a rather high abundance. If we ignore the question of 'formation interval' ( $\left.\Delta_{2, \text { UOC }}\right)$, then one set of results points to a value of $\sim 10^{-7}$ and the other to $\sim 10^{-6}$. The latter value then appears to indicate that the early estimate by Birck \& Lugmair (1988) from just the ${ }^{60} \mathrm{Ni}$ anomaly in CAIs was approximately correct. The difference between the two values is so large as to make a firm conclusion about the AGB model very difficult. If the true value of $\left({ }^{60} \mathrm{Fe} /{ }^{56} \mathrm{Fe}\right)_{\Delta_{1}}$ is approximately $10^{-7}$, then a SN source would be excluded, not only according to Rauscher et al. (2002) models, but also by adopting previous nucleosynthesis predictions by Woosley \& Weaver $(1995)$. For $\left({ }^{60} \mathrm{Fe} /{ }^{56} \mathrm{Fe}\right)_{\Delta_{1}}$ of approximately $(1-3) \times 10^{-7}$ and $f_{0}$ of about $(0.5-1) \times 10^{-2}$, it is then required that the contaminating source be an AGB star less massive than $3.0 \mathrm{M}_{\odot}$, if $Z \approx 0.02$. Obtaining values of $\left({ }^{60} \mathrm{Fe} /{ }^{56} \mathrm{Fe}\right)_{\Delta_{1}}$ of $\sim 10^{-6}$ would require an AGB source of $M \gtrsim 3 \mathrm{M}_{\odot}$ and/or $Z$ well below $\mathrm{Z}_{\odot}$. We note that for $M>3 \mathrm{M}_{\odot}$, there would be a serious conflict matching ${ }^{60} \mathrm{Fe} /{ }^{56} \mathrm{Fe}$ with the ${ }^{26} \mathrm{Al} /{ }^{27} \mathrm{Al},{ }^{41} \mathrm{Ca} /{ }^{40} \mathrm{Ca}$ and ${ }^{107} \mathrm{Pd} /{ }^{108} \mathrm{Pd}$ results. An AGB star with $M$ of $2-3 \mathrm{M}_{\odot}$ still appears to be the most promising possibility, although, as we have mentioned, present models (which do not include cool bottom processes) would suffer from a shortage of ${ }^{26} \mathrm{Al}$ by a factor of about 3 . If cool bottom processes could operate in this mass range, then ${ }^{60} \mathrm{Fe}$ at levels of $\left({ }^{60} \mathrm{Fe} /{ }^{56} \mathrm{Fe}\right)_{\Delta_{1}} \approx 3 \times 10^{-7}$ might be explained together with ${ }^{107} \mathrm{Pd}$ and ${ }^{26} \mathrm{Al}$ in this source.

As noted earlier by Wasserburg et al. (1998), for high ${ }^{60} \mathrm{Fe} /{ }^{56} \mathrm{Fe}$ values there should be large isotopic shifts in ESS materials with modest $\mathrm{Fe} / \mathrm{Ni}$ fractionation. Most recent data have focused on very great enrichments of $\mathrm{Fe}$ relative to $\mathrm{Ni}$ in $\mathrm{FeS}$ using ion probe techniques at low precision. However, a more definitive test could be done on mineral phases in equilibrium formed from a melt. The first efforts by Shukolyukov \& Lugmair (1993) sought effects in eucrites that are Ni-deficient due to the apparent separation of an $\mathrm{Fe}-\mathrm{Ni}$ core on their parent planet. However, it is possible that liquid droplets such as unmetamorphosed chondrules would show sufficient fractionation between $\mathrm{Fe}$ and $\mathrm{Ni}$ (for example, between olivines and pyroxenes and metal) so that high precision $(0.5 \epsilon \mathrm{u}$, where $1 \epsilon \mathrm{U}=1$ part per ten thousand) Thermal Ionisation Mass Spectrometry (TIMS), or Ion Coupled Plasma Mass Spectrometry (ICPMS) methods could be used to establish isochrons. This would permit measurements on phases with very different paragenesis than FeS and $\mathrm{FeO}$ samples. An enrichment factor of 7.5 would yield a $5 \epsilon \mathrm{u}$ effect at ${ }^{60} \mathrm{Fe} /{ }^{56} \mathrm{Fe} \simeq 10^{-6}$. Internal ${ }^{60} \mathrm{Fe}-$ ${ }^{60} \mathrm{Ni}$ isochrons in such samples would greatly clarify the matter. 


\section{Conclusions}

In this paper we have reviewed the studies on the origin of ESS short-lived radioactivities, after a brief account of the relevant measurements and of the hypotheses previously presented for their interpretation. It now appears that all nuclei with meanlife in excess of $10 \mathrm{Myr}$ can be explained by the continuous injection of supernova debris into the ISM, where a quasi-steady state between production and decay could be established. This assumes that two (or more) $r$-process sources are dominantly producing the heavy $r$-nuclei. In addition, ${ }^{53} \mathrm{Mn}$ (mean life 5.3 Myr) can also be produced by long-term galactic nucleosynthesis. Other nuclei with a short mean life, from ${ }^{107} \mathrm{Pd}$ $(\bar{\tau}=9.4 \mathrm{Myr})$ down to ${ }^{41} \mathrm{Ca}(\bar{\tau}=0.15 \mathrm{Myr})$, all require a late contamination by a stellar source, with the exception of ${ }^{10} \mathrm{Be}$. Presently available chemical yields from massive star evolution and explosion indicate that a nearby supernova source does not appear capable of accounting for the ESS concentration of ${ }^{26} \mathrm{Al},{ }^{41} \mathrm{Ca}$ and ${ }^{107} \mathrm{Pd}$, without exceedingly overproducing ${ }^{53} \mathrm{Mn}$ and probably also ${ }^{60} \mathrm{Fe}$. A much better situation is provided by nucleosynthesis models for low mass AGB stars undergoing marginal $s$-processing and cool bottom processing, as might be the case for stars below $3 \mathrm{M}_{\odot}$. A contamination by such a source naturally accounts for the measured ESS concentrations of the three isotopes above and also gives estimates for ${ }^{60} \mathrm{Fe}$ within the present experimental uncertainty, for reasonable choices of the ages of meteoritic samples in which measurements of short-lived nuclei are available. The critical issue for ${ }^{26} \mathrm{Al}$ is the extent to which cool bottom processes can be effective in stars of masses between $2.0 \mathrm{M}_{\odot}$ and $3.0 \mathrm{M}_{\odot}$. This issue is not yet resolved.

As a concluding remark we want to stress that in some cases the abundances of short-lived nuclei in different samples of early solar system material are not related in a simple self-consistent pattern. This applies, for example, to ${ }^{107} \mathrm{Pd}$ or to ${ }^{53} \mathrm{Mn}$ abundances in different samples and can be seen in numerous examples where isochrons of good quality are obtained but the results are not in accord with the usually inferred 'times of formation'. Some samples with 'primitive' abundances appear to be younger by large amounts $\left(\Delta_{2} \sim 16 \mathrm{Myr}\right)$ and, alternatively, there are planetary differentiates that are thought to be 'younger' but have unusually high abundances of, say, ${ }^{53}$ Mn (see also Hutcheon et al. 1992, 1998; Lugmair \& Skukolyukov 1998; Chen \& Wasserburg 1996). In parallel with improved efforts for modeling the production of short-lived nuclei in stars and locally, also more intense and careful experimental attempts are needed, comparing the abundances of different short-lived nuclei in the same objects and pursuing a better insight into the possible mechanisms of resetting.

\section{Acknowledgments}

We all wish to thank John Lattanzio for his generous share of information, data, advice and good wines. Suggestions of critical importance and stimulating comments were provided by G. Lugmair, E. Zinner, J. José, O. Straniero, M. Limongi and A. Chieffi. In particular, we would like to acknowledge the extremely helpful and detailed comments and criticisms by G. Huss that greatly aided in improving this report. Cynthia A. Craig reminded us old Italian aphorisms, which are useful in a wide variety of occasions. M.B. and R.G. acknowledge support from the Italian MIUR-FIRB Project $\mathrm{M}_{\odot}$ 'Astrophysical Origin of Heavy Elements beyond Fe'. G.J.W. acknowledges support from NASA NAGS-11725. Caltech Division Contribution 8905(1107).

\section{References}

Abia, C., Domínguez, I., Gallino, R., Busso, M., Masera, S., Straniero, O., de Laverny, P., Plez, B., \& Isern, J. 2002, ApJ, 579,817

Alexander, E. C. Jr, Lewis, R. S., Reynolds, J. H., \& Michel, M. C. 1971, Science, 172, 837

Allègre, C. J., Manhès, G., \& Göpel, C. 1995, Geochim. Cosmochim. Acta, 59, 1445

Amelin, Y., Krot, A. N., Hutcheon, I. D., \& Ulyanov, A. A. 2002, Science, 297, 1678

Arnould, M., Paulus, G., \& Meynet, G. 1997, A\&A, 321, 452

Arnould, M., \& Prantzos, N. 1999, New Astronomy, 4, 283

Bahcall, J., Pinsonneault, M. H., \& Wasserburg, G. J. 1995, Rev. Mod. Phys., 67, 781

Bao, Z. Y., Beer, H., Käppeler, F., Voss, F., Wisshak, K., \& Rauscher, T. 2000, Atom. Data Nucl. Data Tables, 76, 70

Birck, J.-L., \& Allègre, C. A. 1985, Geophys. Res. Lett., 12, 745

Birck, J.-L., \& Lugmair, G. 1988, Earth Planet. Sci. Lett., 90, 131

Brazzle, R. H., Pravdivtseva, O. V., Meshik, A. P., \& Hohenberg, C. M. 1999, Geochim. Cosmochim. Acta, 63, 739

Busso, M., Gallino, R., Lambert, D. L., Travaglio, C., \& Smith, V. V. 2001, ApJ, 557, 802

Busso, M., Gallino, R., \& Wasserburg, G. J. 1999, ARA\&A, 37, 239

Cameron, A. G. W. 1960, AJ, 65, 485

Cameron, A. G. W. 1993, in Protostars and Planets III, ed. E. H. Levy, J. L. Lunine (Tucson: Univ. of Arizona Press), 47

Cameron, A. G. W. 2001, ApJ, 562, 456

Cameron, A. G. W., Höflich, P., Myers, P. C., \& Clayton, D. D. 1995, ApJ, 447, L53

Cameron, A. G. W., Thielemann, F.-K., \& Cowan, J. J. 1993, Phys. Rep., 227, 283

Cameron, A. G. W. \& Truran, J. W. 1977, Icarus, 30, 447

Cameron, A. G. W., Vanhala H., \& Höflich P. 1997, in Astrophysical Implications of the Laboratory Study of Presolar Materials, ed. T. J. Bernatowicz, \& E. Zinner, AIP Conf. Proc., 402 (Woodbury, New York: AIP), 665

Cantelaube, Y., Maurette, H., \& Pellas, P. 1967, in Radioactive Dating and Methods of Low Level Counting (Vienna: Int. Atomic Energy), 215

Carlson, R. W., \& Hauri, E. H. 2001, Geochim. Cosmochim. Acta, 65, 1839

Chen, J. H., Papanastassiou, D. A., \& Wasserburg, G. J. 1996, in Earth Processes: Reading the Isotopic Code, ed. A. Basu, \& S. Hart, Geophys. Monograph 95 (Washington: Amer. Geophys. U.), 1

Chen, J. H., Papanastassiou, D. A., \& Wasserburg, G. J. 2003, Lunar Planet. Sci., 34, 1789

Chen, J. H., \& Wasserburg, G. J. 1981a, Analit. Chem., 53, 2060

Chen, J. H., \& Wasserburg, G. J. 1981b, Earth Planet. Sci. Lett., 52, 1

Chieffi, A., \& Limongi, M. 2003, ApJ, in press (astroph/0304185)

Clayton, R. N. 2002, Nature, 415, 860

Clayton, R. N., Grossman, L., \& Mayeda, T. K. 1973, Science, 182,485 
Clayton, R. N., Mayeda, T. K., \& Onuma, N. 1976, Earth Planet. Sci. Lett., 30, 10

Cohen, J. G., Christlieb, N., Qian, Y.-Z., \& Wasserburg, G. J. 2003, ApJ, 588, 1082

Cowan, J. J., Pfeiffer, B., Kratz, K. L., Thielemann, F. K., Sneden, C., Burles, S., Tytler, D., \& Beers, T. C. 1999, ApJ, 521, 194

Desch, S. J., Srinivasan, G., \& Connolly, H. C. Jr 2003, Lunar Planet. Sci., 34, 1394

Diehl, R., \& Timmes, F. X. 1996, PASP, 110, 637

Fish, R. A., Goles, G. G., \& Anders, E. 1960, ApJ, 132, 243

Gallino, R., Arlandini, C., Busso, M., Lugaro, M., Travaglio, C., Straniero, O., Chieffi, A., \& Limongi, M. 1998, ApJ, 497, 338

Gallino, R., Raiteri, C. M., \& Busso, M. 1993, ApJ, 410, 400

Goswami, J. N., \& Vanhala, H. A. T. 2000, in Protostars and Planets IV, ed. V. Mannings, A. P. Boss, \& S. S. Russell (Tucson: Univ. Arizona Press), 963

Gounelle, M., Shu, F. H., Shang, H., Glassgold, A. E., Rehm, K. E., \& Lee, T. 2001, ApJ, 548, 1051

Harper, C. L., \& Jacobsen, S. B. 1994, Lun. Plan. Sci., 25, 509

Harper, C. L., \& Jacobsen, S. B. 1996, Geochim. Cosmochim. Acta, 60,1131

Hsu, W., Wasserburg, G. J., \& Huss, G. R. 2000, Earth Planet. Sci. Lett., 182,15

Hudson, G. B., Kennedy, B. M., Podosek, F. A., \& Hohenberg, C. M. 1989, Lunar Planet. Sci., 19, 547

Hutcheon, I. D., Olsen, E., Zipfel, J., \& Wasserburg, G. J. 1992, Lunar Planet. Sci. Conf. XXIII, 565

Hutcheon, I. D., Krot, A. N., Keil, K., Phinney, D. L., \& Scott, E. R. D. 1998, Meteorit. Planet. Sci., 33, A72

Iliadis, C., Champagne, A., José, J., Starrfield, S., Tupper, P., et al. 2002, ApJS, 142, 105

Jacobsen, S. B., \& Wasserburg G. J. 1984, Earth. Planet. Sci. Lett., 67,137

Jeffery, P. M., \& Reynolds. J. H. 1961, J. Geophys. Res., 66, 3582

Kastner, J. H., \& Myers, P. C. 1994, ApJ, 421, 605

Kelly, W. R., \& Wasserburg, G. J. 1978, Geophys. Res. Lett., 5, 1079

Kleine, T., Münker, C., Mezger, K., \& Palme, H. 2002, Nature, $418,952$.

Knödlseder, J. 1999, ApJ, 510, 915

Kothes, R., Uyaniker, B., \& Pineault, S. 2001, ApJ, 560, 236

LaTourrette, T., \& Wasserburg, G. J. 1997, Lunar Planet. Sci., 28, 781

Lattanzio, J. C., \& Forestini, M. 1999, in Asymptotic Giant Branch Stars, IAU Symp. 191, ed. T. Le Bertre, A. Lèbre, \& C. Waelkens (San Francisco: ASP), 31

Lee, D.-C., \& Halliday, A. N. 1995, Nature, 378, 771

Lee, D.-C., \& Halliday, A. N. 1996, Science, 274, 1876

Lee, D.-C., \& Halliday, A. N. 1997, Nature, 388, 854

Lee, D.-C., \& Halliday, A. N. 1998, Lunar Planet. Sci., 29, 1416

Lee, T., Papanastassiou, D. A., \& Wasserburg, G. J. 1976, Geophys. Res. Lett., 3, 109

Lee, T., Papanastassiou, D. A., \& Wasserburg, G. J. 1977, ApJ, 211, L107

Lee, T., Shu, F. H., Shang, H., Glassgold, A. E., \& Rehm, K. E. 1998, ApJ, 506, 898

Lentz, E. J., Branch, D., \& Baron, E. 1999, ApJ, 512, 678

Lugaro, M., Karakas, A., Champagne, A., Lattanzio, J. C., \& Cannon, R. 2001, Mem. Soc. Astrom. It., 72, 319

Lugmair, G. W., \& Galer, S. J. G. 1992, Geochim. Cosmochim. Acta, 56,1673

Lugmair, G. W., \& Marti, K. 1977, Earth Planet. Sci. Lett., 35, 273

Lugmair, G., Shimamura, T., Lewis, R. S., \& Anders, E. 1983, Science, 222, 1015

Lugmair, G. W., \& Shukolyukov, A. 1998, Geochim. Cosmochim. Acta, 62, 2863

Lugmair, G. W., Shukolyukov, A., \& MacIsaac, Ch. 1995, in Nuclei in the Cosmos III, ed. M. Busso, R. Gallino, \& C. M. Raiteri (New York: AIP), 591

MacPherson, G. J., Davis, A. M., \& Zinner, E. K. 1995, Meteoritics, 30,365
MacPherson, G. J., \& Huss, G. R. 2001, Lunar Planet. Sci., 32, 1882 MacPherson, G. J., Huss, G. R. \& Davis, A. M. 2003, Geochim. Cosmochim. Acta, 67, (in press)

Mahoney, W. A., Ling, J. C., Wheaton, W. A., \& Jacobson, A. S. 1984, ApJ, 286, 578

Marhas, K. K., Goswami, J. N., \& Davis, A. M. 2002, Science, 298, 2182

Marhas, K. K., Hutcheon, I. D., Krot, A. N., Goswami, J. N., \& Komatsu, M. 2000, Meteorit. Planet. Sci., 35, A102

McCulloch, M. T., \& Wasserburg, G. J. 1978, ApJ, 220, L15

McKeegan, K. D., Chaussidon, M., \& Robert, F. 2000, Science, 289, 1334

Merrill, P. W. 1952, Science, 115, 484

Meyer, B. S., \& Clayton, D. D. 2000, Space Sci. Rev., 92, 133

Meynet, G., Arnould, M., Prantzos, N., \& Paulus, G. 1997, A\&A, 320,460

Mostefaoui, S., Lugmair, G. W., Hoppe, P., \& El Goresy, A. 2003, Lunar Planet. Sci., 34, 1585

Mowlavi, N., \& Meynet, G. 2000, A\&A, 361, 959

Murty, S. V. S., Goswami, J. N., \& Shukolyukov, Y. A. 1997, ApJ, 475, L65

Naya, J. E., Barthelmy, S. D., Bartlett, L. M., Gehrels, N., Parsons, A. Teegarden, B. J., Tueller, J., Leventhal, M., et al. 1998, ApJ, 499, L169

Nollett, K. M., Busso, M., \& Wasserburg, G. J. 2003, ApJ, 582, 1036

Pagel, B. E. 1997, Nucleosynthesis and Chemical Evolution of Galaxies, Cambridge Univ. Press: Cambridge

Palla, F., \& Stahler, S. W. 2000, ApJ, 540, 255

Podosek, F. A., \& Lewis, R. S. 1972, Earth Planet. Sci. Lett., 15, 101

Podosek, F. A., \& Nichols, R. H. Jr 1997, in Astrophysical Implications of the Laboratory Study of Presolar Materials, AIP Conf. Proc. 402, ed. T. J. Bernatowicz, E. Zinner (Woodbury, New York: AIP), 617

Podosek, F. A., \& Swindle, T. D. 1988, in Meteorites and the Early Solar System, ed. J. F. Kerridge, M. S. Matthews (Tucson: University of Arizona Press), 1093

Prantzos, N., \& Diehl, R. 1996, Phys. Rep., 267, 1

Preibisch, T., \& Zinnecker, H. 1999, AJ, 117, 338

Qian, Y.-Z., Vogel, P., \& Wasserburg, G. J. 1998a, ApJ, 494, 285

Qian, Y.-Z., Vogel, P., \& Wasserburg, G. J. 1998b, ApJ, 506, 868

Qian, Y.-Z., \& Wasserburg, G. J. 2002, ApJ, 567, 515

Qian, Y.-Z., \& Wasserburg, G. J. 2003, ApJ, 588, 1099

Rauscher, T., Heger, A., Hoffman, R. D., \& Woosley, S. E. 2002, ApJ, 576, 323

Rauscher, T., \& Thielemann, F.-K. 2000, Atomic Data Nucl. Data Tables, 75,1

Reimers, D. 1975, in Problems in Stellar Atmospheres and Envelopes, ed. B. Baschek, W. H. Kegal, \& G. Traving (Berlin: Springer), 229

Reynolds, J. H. 1960, Phys. Rev. Lett., 4, 8

Rotaru, M., Birck, J.-L., \& Allègre C. J. 1992, Nature, 358, 465

Rowe, M. W., \& Kuroda, P. K. 1965, J. Geophys. Res., 70, 709

Russell, S. S., Srinivasan, G., Huss, G. R., Wasserburg, G. J., \& MacPherson G. J. 1996, Science, 273, 757

Sahijpal, S., Goswami, J. N., \& Davis, A. M. 2000, Geochim. Cosmochim. Acta, 64, 1989

Sahijpal, S., Goswami, J. N., Davis, A. M., Grossman, L., \& Lewis, R. S. 1998, Nature, 391, 559

Sahijpal, S., \& Soni, P. 2003, Lunar Planet. Sci. 34, 1087

Scheffler, H., \& Elsässer, H. 1987, Physics of the Galaxy and Interstellar Matter (Berlin \& New York: Springer-Verlag)

Schramm, D. D., Tera, F., \& Wasserburg, G. J. 1970, Earth Planet. Sci. Lett., 10, 44

Schramm, D. N., \& Wasserburg, G. J. 1970, ApJ, 162, 57

Shu, F. H., Shang, H., Glassgold, A. E., \& Lee, T. 1997, Science, 277,1475

Shu, F. H., Shang, H., Gounelle, M., \& Glassgold, A. E. 2001, ApJ, 548,1051

Shu, F. H., Shang, H., \& Lee, T. 1996, Science, 271, 1545 
Shukolyukov, A., \& Lugmair, G. W. 1993a, Science, 259, 1138 Shukolyukov, A., \& Lugmair, G. W. 1993b, Earth Planet. Sci. Lett., 119,159

Shukolyukov, A., \& Lugmair, G. W. 1996, Meteor. Planet. Sci., 31, A129

Simanton, J. R., Rightmire, R. A., Long, A. L., \& Kohman, T. P. 1954, Phys. Rev., 96(6), 1711.

Sneden, C., Cowan, J. J., Burris, D. L., \& Truran, J. W. 1998, ApJ, 496, 235

Sneden, C., Cowan, J. J., Ivans, I. I., Fuller, G. M., Burles, S., Beers, T. C., \& Lawler, J. E. 2000, ApJ, 533, L139

Srinivasan, G., Sahijpal, S., Ulyanov, A. A., \& Goswami, G. N. 1996, Geochim. Cosmochim. Acta, 60, 1823

Srinivasan, G., Ulyanov, A. A., \& Goswami, J. N. 1994, ApJ, 431, L67

Stanke, T., McCaughrean, M. J., \& Zinnecker, H. 2002, A\&A, 392, 239

Starrfield, S., Sparks, W. M., Truran, J. W., \& Wiescher, M. C. 2000, ApJS, 127, 485

Stewart, B., Papanastassiou, D. A., \& Wasserburg, G. J. 1994, Geochim. Cosmochim. Acta, 58, 3487

Stewart, B., Papanastassiou, D. A., \& Wasserburg, G. J. 1996, Earth Planet. Sci. Lett., 143, 1

Storzer, D., \& Pellas, P. 1977, Earth Planet. Sci. Lett., 35, 285

Sturner, S. J., \& Naya, J. E. 1999, ApJ, 526, 200

Straniero, O., Chieffi, A., Limongi, M., Busso, M., Gallino, R., \& Arlandini, C. 1997, ApJ, 478, 332

Tachibana, S., \& Huss, G. R. 2003a, ApJ, 558, L41

Tachibana, S., \& Huss, G. R. 2003b, Lunar Planet. Sci., 34, 1737

Tera, F., \& Carlson, R. W. 1999, Geochim. Cosmochim. Acta, 63,1877

Thiemens, M. H. 1999, Science, 283, 341

Thiemens, M. H., \& Heidenreich, J., III, 1983, Science, 219,1073
Timmes, F. X., Woosley, S. E., Hartmann, D. H., Hoffmann, R. D., Weaver, T. A., \& Matteucci, F. 1995, ApJ, 449, 204

Travaglio, C., Galli, D., Gallino, R., Busso, M., Ferrini, F., \& Straniero, O. 1999, ApJ, 521, 691

Urey, H. C. 1955, Proc. Nat. Acad. Sci. U. S., 41, 127

Urey, H. C., \& Donn, B. 1956, ApJ, 124, 307

Vanhala, H. A. T., \& Boss, A. P. 2000, ApJ, 538, 911

Vanhala, H. A. T., \& Boss, A. P. 2002, ApJ, 575, 1144

Wasserburg, G. J. 1987, Earth Planet. Sci. Lett., 86, 129

Wasserburg, G. J., \& Arnould, M. 1987, in Nuclear Astrophysics, eds. W. Hillebrandt, R. Kuhfuss, E. Müller \& J. W. Truran (Berlin: Springer Verlag), 262

Wasserburg, G. J., Busso, M., \& Gallino, R. 1996, ApJ, 466, L109

Wasserburg, G. J., Busso, M., Gallino, R., \& Raiteri, C. M. 1994, ApJ, 424, 412

Wasserburg, G. J., Fowler, W. A., \& Hoyle, F. 1960, Phys. Rev. Lett., 4, 112

Wasserburg, G. J., Gallino, R., \& Busso, M. 1998, ApJ, 500, L189

Wasserburg, G. J., Gallino, R., Busso, M., Goswami, J. N., \& Raiteri, C. M. 1995, ApJ, 440, L101

Wasserburg, G. J., Huneke, J. C., \& Burnett, D. S. 1969a, Phys. Rev. Lett., 22, 1198

Wasserburg, G. J., Huneke, J. C., \& Burnett, D. S. 1969b, J. Geophys. Res., 74, 4221

Woosley, S. E., \& Weaver, T. A. 1995, ApJS, 101, 181

Woosley, S. E., Fowler, W. A., Holmes, J. A., \& Zimmerman, B. A. 1978, Atomic Data Nucl. Data Tables, 22, 371

Yin, Q., Jacobsen, S. B., Yamashita, K., Blichert-Toft, J., Télouk, P., \& Albarède, F. 2002, Nature, 418, 949

Yokoi, K., Takahashi, Y., \& Arnould, M. 1985, A\&A, 145, 339

Zinnecker, H. 2002, Astrophys. Space Sci., 281, 147

Zinner, E. 1998, Ann. Rev. Earth Planet. Sci., 26, 147

Zinner, E., \& Göpel, C. 2002, Meteorit. Planet. Sci., 37, 1001 